\title{
Kinetics of dislocation emission in 3D: from the crack toward the free surface
}

\author{
P. Hora, A. Machová*, J. Červ, A. Uhnáková \\ Institute of Thermomechanics AS CR v.v.i., Dolejskova 5, 18200 Prague 8, Czech Republic
}

Received 9 August 2018, received in revised form 22 November 2018, accepted 5 February 2019

\begin{abstract}
The kinetics of dislocations emitted from a crack is studied via molecular dynamics (MD) in a $3 \mathrm{D}$ bcc iron crystal. The atomistic results show that edge dislocation segments in the middle of the crystal accelerate at the nearest vicinity of the free crystal surface. The dislocations in MD penetrate the surface layers in transonic or supersonic regimes. The possible sources for such behavior are discussed in the framework of continuum models and using stress calculations on the atomistic level. Acoustic emission patterns arising from the fast dislocation motion in MD are visualized via the local kinetic energies of individual atoms and further modeled as a moving source of the stress waves in the anisotropic continuum.
\end{abstract}

K e y w or d s: edge crack, dislocation emission, free surface, acoustic emission, bcc iron

\section{Introduction}

Atomistic simulations can verify continuum models or bring new predictions to materials science or another field of interest.

It is well known that dislocations are attracted to the free sample surface since the disappearance of dislocations via formation of surface steps enables a decrease in the potential (strain) energy in the system. Koehler [1] introduced a simplified continuum model, where the process can be modeled by means of a mirror dislocation of the opposite sign, lying in the same slip plane on the opposite side of the free surface at the same distance $R$. The driving force is proportional to $1 / R$ and, in the static continuum models, it is called as a mirror or image force acting on the dislocation toward the free surface.

Recent solutions of the elastic stress field of an injected and uniformly moving dislocation toward the free surface enabled the derivation of analytical expressions for the elasto-dynamic image force [2] in the isotropic continuum, also depending on a relative time scale and the velocity of uniformly moving dislocation. These image forces may lead to an acceleration of dislocation motion toward the free surface. The limiting velocity of dislocations at the free surface in the isotropic continuum is the velocity of surface Rayleigh waves [2] and, in the atomistic lattice, it should be the velocity of sound by the known Frenkel-Kontorova linear atomic spring model [3].

Another possible source for acceleration of dislocations toward the free surface is the surface stress, which is a new aspect (approach) presented in the paper. There are missing interatomic interactions at the free surfaces of crystals in the normal direction to the surface. So the free surfaces relax using the change in interplanar distances to minimize the potential energy in the system and to eliminate the unresolved normal forces at the free surface.

Surface relaxation and surface energies are real, experimentally measurable quantities. In continuum language, after the surface relaxation, the normal stress components at the free surfaces vanish, but the other stress components along the free surface need not be zero after the surface relaxation, and they are called as surface stress or surface tension (as in a drop of water).

The models mentioned above are treated or discussed in the present study to explain the acceleration of dislocations toward the free surface, observed in our free 3D atomistic simulations by molecular dynamic (MD) technique.

*Corresponding author: e-mail address: machova@it.cas.cz 
This unpublished MD study uses the boundary condition with prescribed displacements at the borders and is devoted to the kinetics of dislocations emitted from the relatively long edge crack embedded in the $3 \mathrm{D}$ rectangular atomistic sample loaded in mode I. The influence of the near right free sample surface on dislocation speed and acoustic emission is studied. MD simulations are completed with stress calculations on the atomistic level [4].

Similar to our previous work, we use an N-body potential [5] for bcc iron (i.e., the description of the Fe-Fe interaction from Table 2 in [5]), successfully tested in many applications. Under the constant displacement conditions, the studied long edge crack (110)[110] (crack plane/crack front) emits dislocations on the $<111>\{112\}$ slip systems (Fig. 1) independent of the loading rate. These dislocation emissions make the crack more stable. The boundary condition used is suitable for the studies of dislocation kinetics, since the process is also well pronounced in the global energy balance, in particular under a relatively slow loading rate. Our question is, why do these dislocations accelerate to the transonic regime at the free crystal surface?

Several theoretical and atomistic studies of the fast dislocation motion above the velocity of the transversal (shear) waves $\left(C_{\mathrm{T}}\right)$ and longitudinal $\left(C_{\mathrm{L}}\right)$ waves have been published, e.g., in [6-11]. The studies mentioned reveal that, under special circumstances, the dislocations can overcome the limiting velocity given by the Frenkel-Kontorova model that has been accepted for a long time [3].

Further development of the models concerning fast dislocations is discussed in Sec. 3 in connection with the results presented.

To the authors' knowledge, no atomistic study concerning the influence of the surface stress on the fast dislocation motion and acoustic emission has been published yet. Atomistic study of the problem mentioned above is the topic of our paper.

\section{MD simulations}

A pre-existing through edge crack of the length $a$ is considered, placed in the middle of a rectangular sample of length $L$, width $W$, and thickness $B$ - see Fig. 1 . The relative crack length corresponds to $a / W=0.8$. The sample was loaded by monotonic uni-axial tension (mode I) via prescribing displacements in $L$-direction for six border BW layers at the bottom and the top of the sample. Surface relaxation was performed before the loading, to avoid its influence on crack tip processes.

Newton's equations of motion for individual atoms were solved in all three directions by a central difference method, using time integration step $h=$

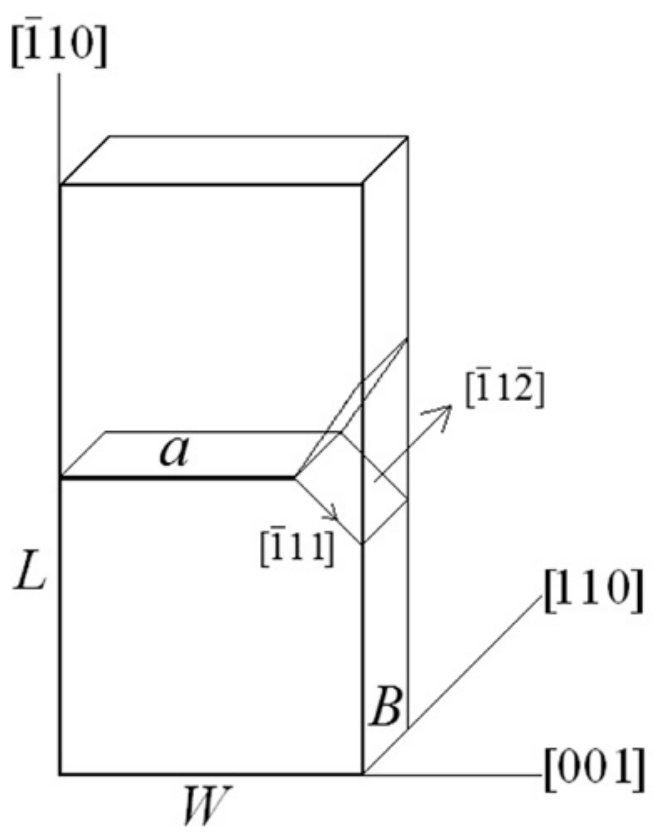

Fig. 1. Sample geometry for crack orientation (110)[110] (crack plane/crack front) and scheme for the available inclined slip systems: the arrow [1111] denotes the slip direction, the arrow $[\overline{1} 1 \overline{2}]$ marks the normal to the lower slip plane $(\overline{1} 1 \overline{2})$ also containing the crack front oriented in the [110] direction. The upper slip system is of orientation $[1 \overline{1} 1](\overline{1} 12)$. The observation planes in MD are the

LW planes (110) perpendicular to the crack front.

$1 \times 10^{-14} \mathrm{~s}$. The surface region mentioned, with prescribed displacements, does not enter the time integration procedure. No periodic boundary conditions were applied in the free 3D atomistic simulations presented.

Atomically sharp edge crack $(\overline{1} 10)[110]$ was created by cutting interatomic bonds across the initial crack plane. The initial temperature was $0 \mathrm{~K}$, and the further thermal atomic motion was not controlled, similar to [10]. The atomistic sample consisted of 440 atomic planes (110) in $L$-direction, 220 planes (001) in $W$ -direction and 30 planes (110) in B-direction along the crack front.

The total number of atoms in the $3 \mathrm{D}$ crystal was $N P O I N=1452$ 000. The atomistic specimen of SEN (single edge notch) type was loaded gradually (linearly in time $t=n h$ ) with prescribed displacements $v(t)$ at the BW surfaces each time step $t=n h$ in the direction [110] along the sample length $L$, i.e., in mode I.

Similar to our previous work, each time step $n$, the total potential $E P O T=E P O T(t)-E P O T(0)$ and kinetic energies $E K I N=E K I N(t)-E K I N(0)$ were monitored in the system. The total number of interatomic LINT interactions and the position of the crack front in the middle of the crystal were also monitored at each time step.

Also, the coordinates, the local kinetic energies 
$E_{\text {kin }}(l i)$ and the local numbers of interactions $K N T$ (i.e., coordination numbers) at individual atoms ( $l i)$ were monitored at selected time steps for graphic purposes in 3D. We scan the atomic configurations in all the planes (110) perpendicular to the crack front, as well as in other planes if necessary.

The presented stress calculations on the atomistic level are based on the interplanar concept [4] that well describes the stress distribution, not only under homogeneous deformation but also in the case when straining is inhomogeneous in the range of interatomic interaction. The free surface is an example.

Note that, as mentioned in [4], the definition of the stress in the atomistic lattice is not unique. The concept of the virial stress utilized in [12] includes stress components both from the potential and kinetic energies. The main component, coming from the potential (strain) energy in the system (also called volume stress), is calculated from all the interatomic interactions surrounding an atom in the considered range of the interactions, while the interplanar stress includes only those interactions in one direction perpendicular to a plane.

The virial volume and interplanar concepts produce the same results under homogeneous straining in the range of interatomic interactions. However, they differ at the free relaxed surfaces: the volume stress oscillates at the free surface, while the interplanar stress vanishes in the normal direction to the free surface.

MD simulations themselves do not require the stress. MD works with the nonlinear interatomic forces and displacements. Additional stress calculations serve merely as complementary tools for comparison with continuum models or further treatment of $\mathrm{MD}$ results.

For MD simulations we use our in-house Fortran codes and, for the graphical treatment of MD results, our codes use the commercial computing MATLAB environment.

\section{Results and discussion}

Under the boundary conditions with prescribed displacements, the stress intensity factor $K_{\mathrm{I}}$ and $T$ stress are mutually bounded with the prescribed displacement $v_{\mathrm{A}}$ applied in mode $\mathrm{I}$, via the relations by Fett [13]: $\sigma_{0}=E v_{\mathrm{A}} H$ and $K_{\mathrm{I}}=F_{\mathrm{I}} \sigma_{0}(\pi a)^{1 / 2}$; $T=f_{\mathrm{I}} \sigma_{0}$, where $E$ stands for Young's modulus, $v_{\mathrm{A}}$ is the prescribed displacement at the borders, $H=L / 2$ and $F_{\mathrm{I}}, f_{\mathrm{I}}$ are the boundary correction factors for a given sample geometry $a / W, H / W$ presented in [13]. The crack length in this study corresponds to $a=$ $88 a_{0}$, where $a_{0}=2.8665 \AA$ is the lattice parameter and Young's modulus $E=1 / A 22=2.237 \times 10^{11} \mathrm{~N} \mathrm{~m}^{-2}$ as in $[3,15]$.

Here we mainly present the results for a relatively slow loading rate $\Delta v_{\mathrm{A}}(t) / \Delta t=14.7 \AA / 20000 h$, where $v_{\mathrm{A}}$ is the applied prescribed displacement. The critical prescribed displacement $v_{\mathrm{G}}=7.35 \AA$ (corresponding to the critical Griffith stress intensity $K_{\mathrm{G}}$ ) is reached per $10000 h$. The loading rate may also be expressed via $K_{\mathrm{G}}$ as $\mathrm{d} K_{\mathrm{A}} / \mathrm{d} t=0.010 K_{\mathrm{G}} / \mathrm{ps}$ ( since $100 h=1$ ps). This notation is often used in crack simulations as it enables a comparison between various MD results and also to determine the instantaneous applied stress intensity via the relation $K_{\mathrm{A}}(t)=t *\left(0.01 K_{\mathrm{G}} / \mathrm{ps}\right)$.

Here, $K_{\mathrm{G}}=0.906 \mathrm{MPam}^{1 / 2}$ stands for Griffith stress intensity $[14,15]$ needed theoretically for cleavage crack extension of the crack (110)[110] with the interatomic potential in use. (The rise time $10000 h$ to reach the critical Griffith level is larger by one order than the period of the basic vibrations along the length $L$ of the atomistic sample, which leads in the elastic region of loading to low kinetic energy in the $3 \mathrm{D}$ sample. So, the loading is called as a relatively slow - see the global energy balance below).

Note that $K_{\mathrm{A}}(t)$ represents the applied level of loading at the upper and the lower sample borders in $L$-direction. The instantaneous stress intensity at the crack front at the same time $t$ is lower since the loading waves need some time to travel from the borders to the crack front during the dynamic loading.

The character of the stress field on a plane (110) in the middle (at $B / 2$ ) of the loaded crystal before dislocation generation is shown in Fig. 2 for the loading rate $0.01 K_{\mathrm{G}} / \mathrm{ps}$. The coordination system related to Figs. 1 and 2 is introduced according to fracture mechanics: the axis $y=[\overline{1} 10]$ is perpendicular to crack faces, the axis $x=[001]$ is oriented in the direction of the potential crack extension, and the $z$-axis [110] is parallel to the crack front. The peak stress in Fig. 2 denotes the position of the crack front at $x=88 a_{0}$, i.e., the crack length in Fig. 1 is $a=88 a_{0}$. The component $S_{x}$ vanishes at the right free surface. The maximum stress component under uniaxial tension is the normal stress component $S_{y}$, where also a short range surface tension is visible at the right free surface arising after the surface relaxation, similar to $S_{z}$. (The surface stress is treated in detail below).

If the stress intensity at the crack front reaches the critical value (a material parameter $K_{\text {disl }}$ ), a dislocation emission on a favored slip system may occur [14]. Under nominal tension stress $\sigma_{0}$ from external loading, the shear stress is $\tau=\sigma_{0} \sin \alpha \cos \alpha$, where $\alpha$ is an inclination angle of the slip system concerning the axis of potential crack extension.

In our case the available slip systems (Fig. 1) are $\langle 111\rangle\{112\}, \alpha$ is about $55^{\circ}$, the so-called Schmid factor $\sin \alpha \cos \alpha=0.47$ and $K_{\text {disl }}=0.892 \mathrm{MPa} \mathrm{m}^{1 / 2}$ according to Beltz-Rice model [15] and also including the $T$-stress. In Fig. 2 for time step 10 750, the stress intensity at the nearest atoms in the positions $r=1 a_{0}$ $-3 a_{0}$ from the crack front reaches the value $K_{\text {tip }}=$ 

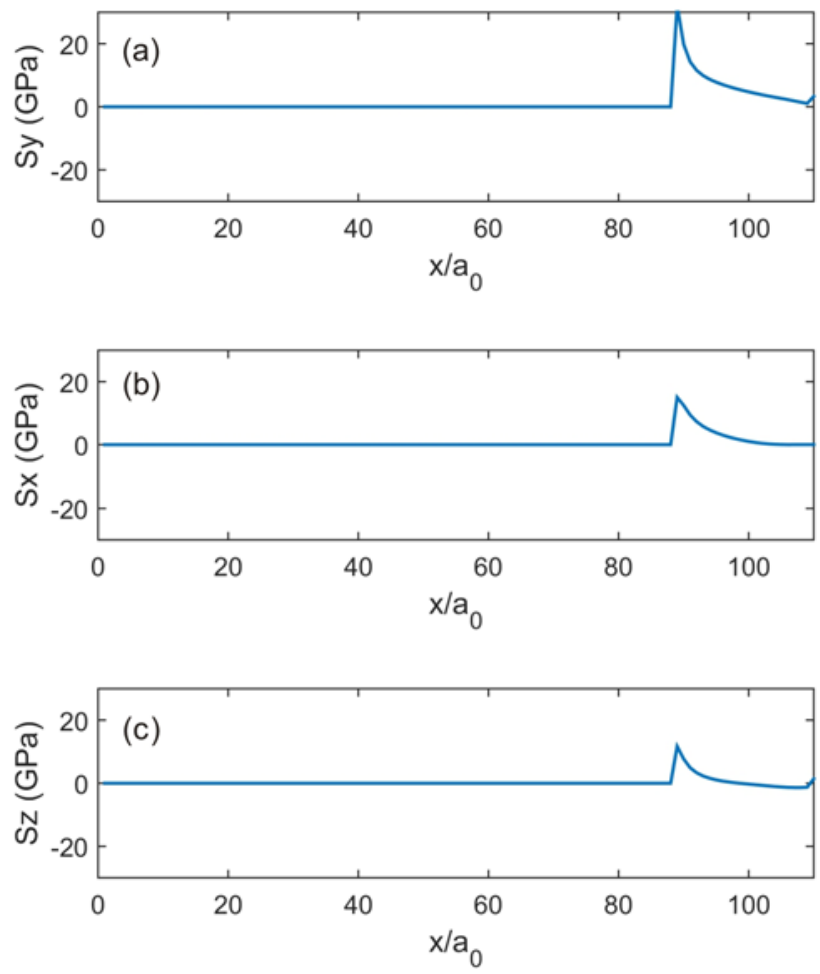

Fig. 2. Stress components in the middle of the sample (at $B / 2$ ) before dislocation generation along the axis of the potential crack extension, time step $10750\left(K_{\mathrm{A}}(t)=\right.$ $\left.1.075 K_{\mathrm{G}}\right)$, the prescribed displacement in the [110] direction corresponds to $v_{\mathrm{A}}(t)=7.9 \AA$ : (a) $S_{y}$; (b) $S_{x}$; (c) $S_{z} ; a_{0}$ is the lattice parameter, the same loading rate $0.010 K_{\mathrm{G}} / \mathrm{ps}$ is used as in Figs. 3-10.

$0.892 \mathrm{MPa} \mathrm{m}^{1 / 2}$ and the average $T$-stress corresponds to $T_{\mathrm{MD}}=-3.865 \mathrm{GPa}$. The evaluation procedure is described, e.g., in [15]. The magnitude of $K_{\text {tip }}$ avoids the prediction from [15], and the sign of the $T$-stress also complies with the continuum treatment in [13] under the boundary condition with prescribed displacements.

Later on, at time step $10760\left(K_{\mathrm{A}}(t)=1.076 K_{\mathrm{G}}\right)$, we detected the first increase in a number of interactions LINT in MD, indicating the beginning of the dislocation generation, followed by subsequent emission, as presented in the next figures. Such behavior is in agreement with the prediction from [15] that negative $T$ supports dislocation emission.

Figure 3 shows the situation on the part of the 3D atomistic sample from Fig. 1, touched by the first dislocation emission under the boundary condition with the prescribed displacements in mode I. Figure 3a illustrates that curved dislocations (red atoms) of mixed character with screw and edge components are emitted in the 3D atomistic sample of finite dimensions. The atoms in Fig. 3a were selected according to the coordination numbers KNT mentioned in Sec. 2.

Here, the dislocation cores (red atoms) have
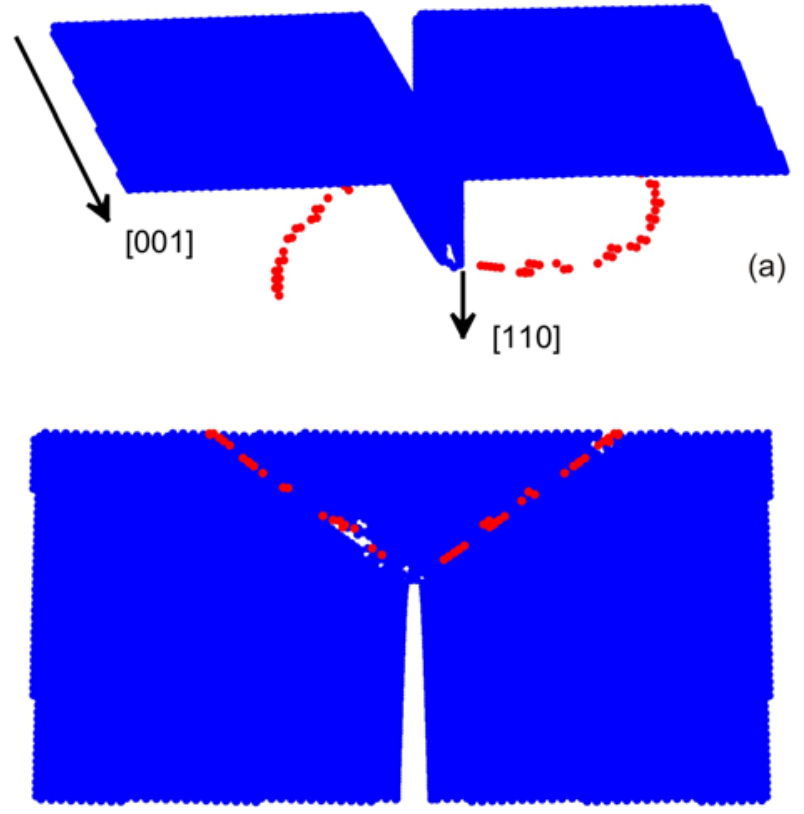

(b)

Fig. 3. (a) 3D visualization of the first emitted dislocations at the edge crack $a / W=0.8$, time step $11861, v_{\mathrm{A}}(t)=$ $8.72 \AA$. Dislocation cores are shown using the red atoms, the crack surfaces and the free sample surfaces are shown via blue atoms. The arrow [001] denotes the axis of the potential crack extension, the arrow [110] shows the orientation of the crack front; (b) projection of the dislocations to LW plane $(110)$, view $(-180,-90)$ in Matlab.

$K N T=16-17$, while at the crack and surface blue atoms $K N T=10$. The regular coordination number in the perfect bcc lattice corresponds to $K N T=14$. (Note that KNT may differ for different interatomic potentials). Figure $3 \mathrm{~b}$ shows the projection of the curved dislocations onto the surface plane LW of the orientation (110). The red atoms (dislocation cores) indicate the slip directions here $\langle 111\rangle$ under an angle of about $55^{\circ}$ concerning the axis of the potential crack growth.

In the middle of the crystal (at $B / 2$ in Fig. 1), plane strain conditions prevail at the crack front with positive stress component $S_{z}>0$ (Fig. 2c). The dislocation segments emitted are of edge character, i.e., with Burgers vector $\boldsymbol{b}=a_{0} / 2\langle 111\rangle$ lying on our (110) observation plane and with the dislocation line $s$ oriented in the perpendicular [110] direction, i.e., along with the crack front in Fig. 3a.

At the free LW surfaces, where plane stress conditions must be observed in the $z$-direction (i.e., $S_{z}=$ $0)$, the screw character $(\boldsymbol{b} \| \boldsymbol{s}$ ) prevails, due to lower strain energy at the free surface. In agreement with continuum models for dislocation emission and crack blunting $[14,15]$, we are focused in this study to edge dislocation segments emitted from the crack front in the middle of the $3 \mathrm{D}$ atomistic sample. 

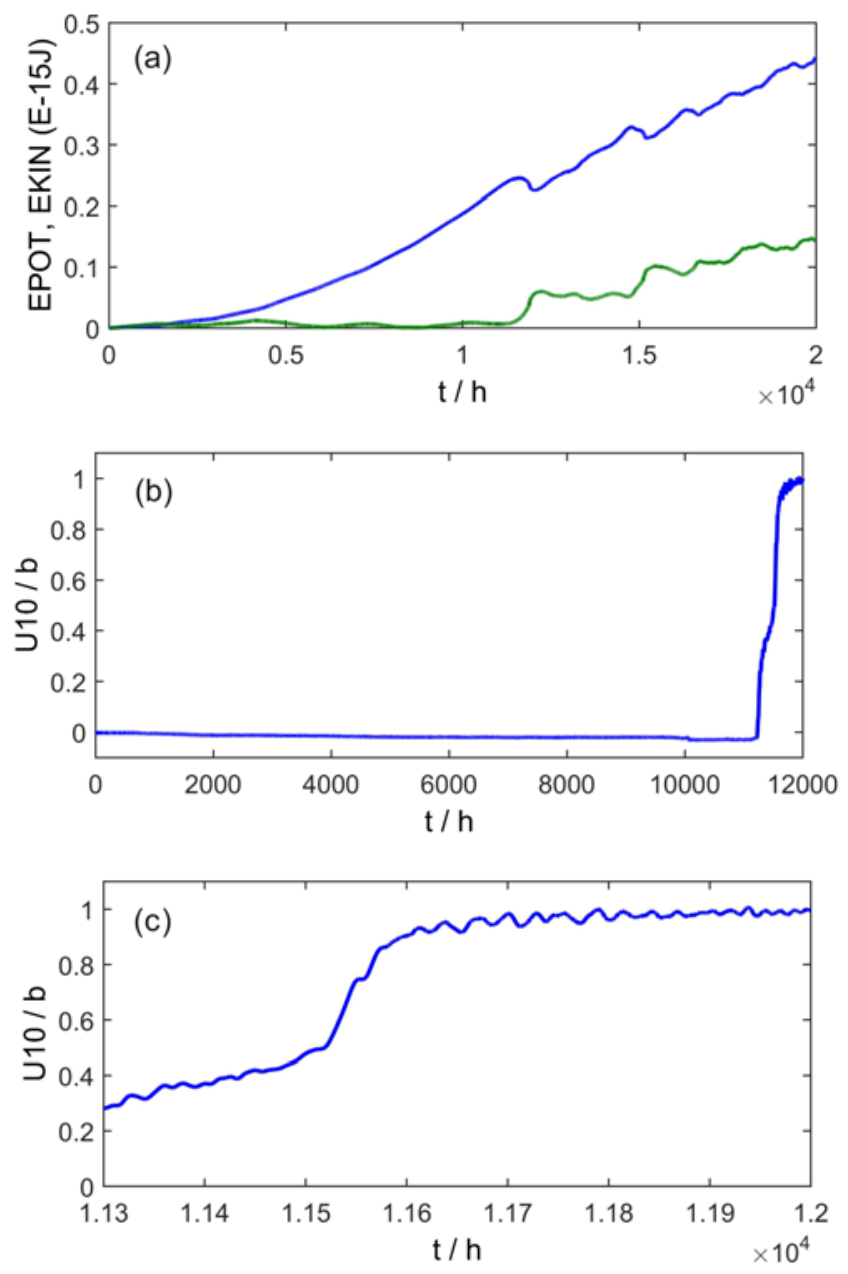

Fig. 4. (a) The global energy balance during multiple dislocation emissions, the first maxima correspond to the first emission, the upper and lower curves correspond to the potential and kinetic energy in the $3 \mathrm{D}$ crystal; (b) time development of the relative shear displacement $U 10 / b$ in the slip system $\langle 111\rangle\{112\}$ during the first dislocation emission in the middle of the 3D bcc iron crystal; (c) a detail from Fig. 4b.

Some atomistic studies utilize periodic boundary conditions along the crack front to model a thick specimen in the $z$-direction. In such a case an emission of straight (line) dislocations from the crack front is expected, similar to plane strain (2D) simulations as in [15]. Here we do not use the periodic conditions in $3 \mathrm{D}$ crack simulations since they do not generally enable free dislocation emission, e.g., from oblique slip systems.

The changes of the total potential and kinetic energies in the atomistic sample, caused by multiple dislocation emissions, are visible well in Fig. 4a under the slow loading rate of $0.010 K_{\mathrm{G}} / \mathrm{ps}$. The first maxima correspond to the first dislocation emission. Further attention is devoted to the first emission.

Figure $4 \mathrm{~b}$ illustrates the generation and the emis- sion of dislocations in the middle layer of the crystal (at $B / 2$ ) using the relative shear displacement $U 10 / b$ at the crack tip in the slip system $\langle 111\rangle\{112\}$. Here, $b=a_{0} \sqrt{3} / 2$ stands for the magnitude of the Burgers vector in bcc iron and $a_{0}$ for the lattice parameter. The data were obtained as the relative shear displacements in the slip direction [111] of two atoms lying at the crack front: one (moving) atom (1) lies at the position $88 a_{0}$ on the lower free crack face (shown in Fig. 5a via the arrow) and the second atom is the "static" neighboring crack tip atom (0) at the position $89 a_{0}$.

Figure 4 also shows that a long time period and high level of external loading at the borders is needed to reach the position $b / 2$ at the crack tip. This occurs at a time step of about $11500\left(K_{\mathrm{A}}(t)=1.15 K_{\mathrm{G}}\right)$ where the global potential energy in Fig. 4a reaches the first maximum. Since the position $b / 2$ is unstable $[14,15]$, the group of atoms lying on the slip plane at the crack tip is displaced (together with the lower part of the crystal) quickly in the direction [111] (like in the block like shear) to a stable position $U 10 / b=1$ under the high shear stress induced by the crack. This occurs at a time step of about 11600 , i.e., at $K_{\mathrm{A}}(t)=$ $1.16 K_{\mathrm{G}}$. A detail from the time development of the relative shear displacement in Fig. 4(c) shows that, in the fastest linear region of the time development, the generation rate reaches a value $V_{\text {gnr }}=b / 3 / \Delta t=$ $206 \mathrm{~m} \mathrm{~s}^{-1}$.

The collective atomic motion causes emission of the complete edge dislocations $\langle 111\rangle\{112\}$ shown in Fig. 5a. It leads to crack blunting and to a significant decrease in the potential energy in the system (see the upper curve in Fig. 4a). The quick transition from the configuration $b / 2$ to $b$ decreases the high shear stress induced by the crack and leads to radiation of the stress waves (acoustic emission (AE)) shown in Fig. 5b. The decrease in stress concentration is shown in Fig. 5c.

The information in Fig. 5b (and as well in Figs. 6b, $7 \mathrm{~b}$ ) is obtained via the map of the local kinetic energies $E_{\text {kin }}(l i)$ of individual atoms $l i$ in the two corresponding middle atomic planes (110) perpendicular to the crack front. The brighter the color, the higher the kinetic energy. The map is scaled as $\operatorname{sqrt}\left(E_{\text {kin }}(l i)\right)$ to see the elastic waves emitted during the emission. The vertical red line in the middle denotes the edge crack. The oblique slip bands starting from the crack tip show the slip system. The value 8 on the color scale corresponds to a maximum of the local kinetic energies in the system.

Further, at the bright dislocation cores $E_{\text {kin }}(l i)=$ $0.33 \times 10^{-20} \mathrm{~J}$, while the average value per atom in the atomistic sample is $E K I N / N P O I N=0.10 \times 10^{-22}$ $\mathrm{J}$, which on the color scale corresponds to 0.01 , i.e., to the black color. In the case of dislocation cores, it is $\sqrt{33}=5.7$, and their color is yellow. Regarding the 


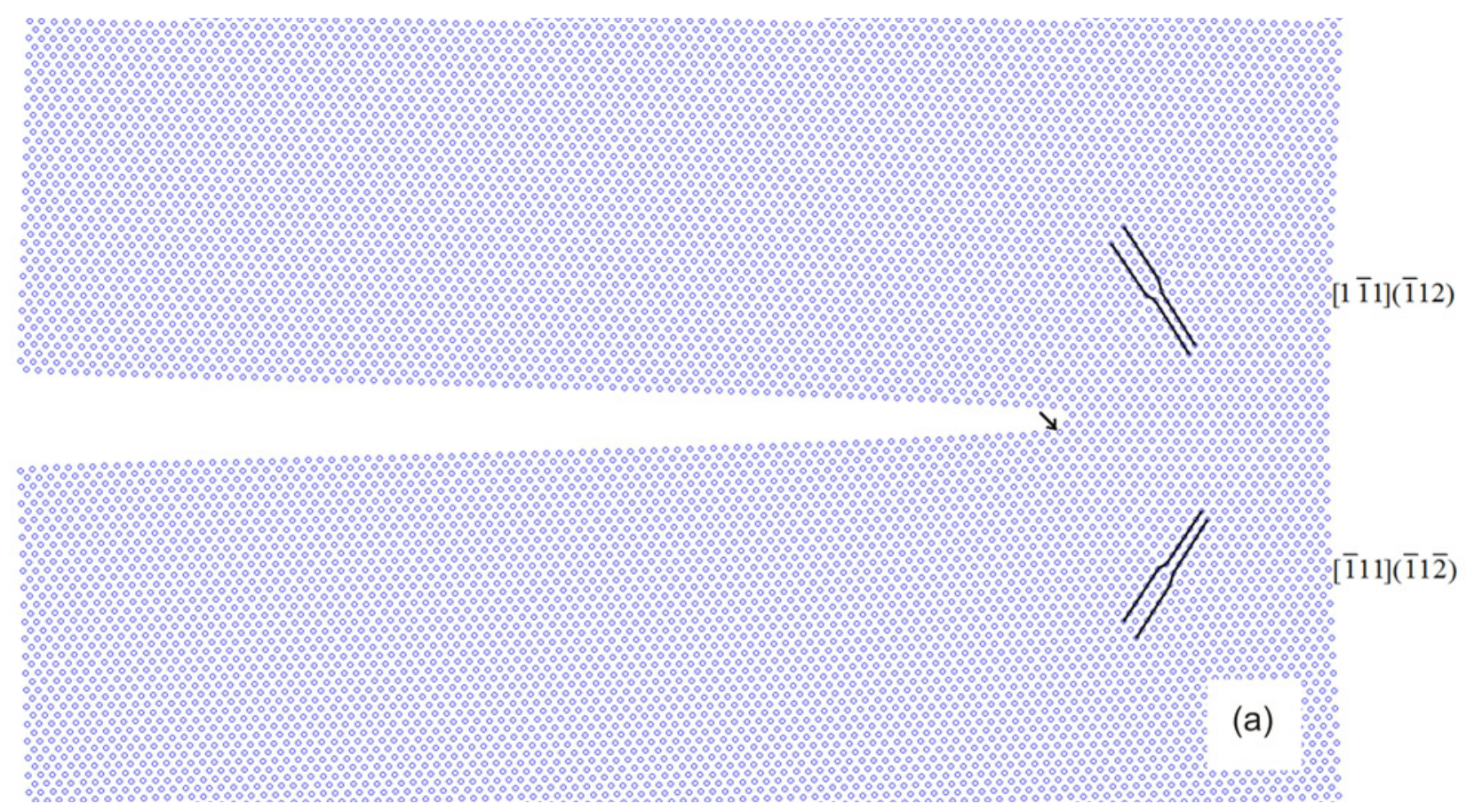

$$
\left(\mathrm{E}_{\mathrm{kin}}\left(\mathrm{I}_{\mathrm{i}}\right) / 10^{-22}\right)^{1 / 2} \text {, double layer=8 }
$$

[001]

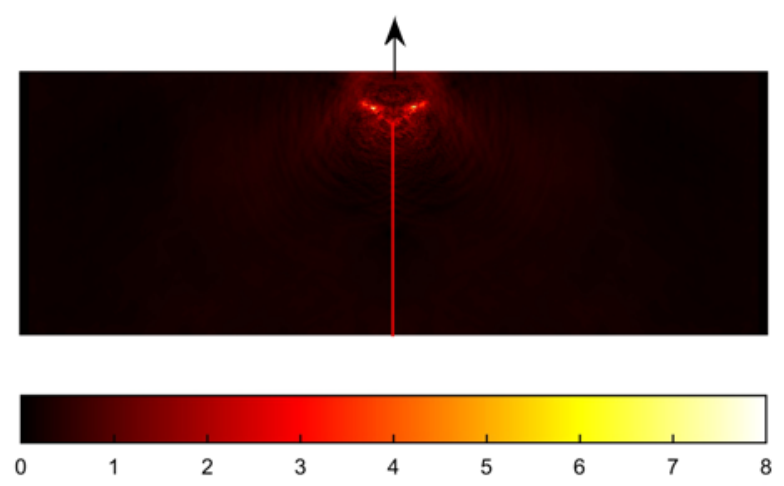

(b)

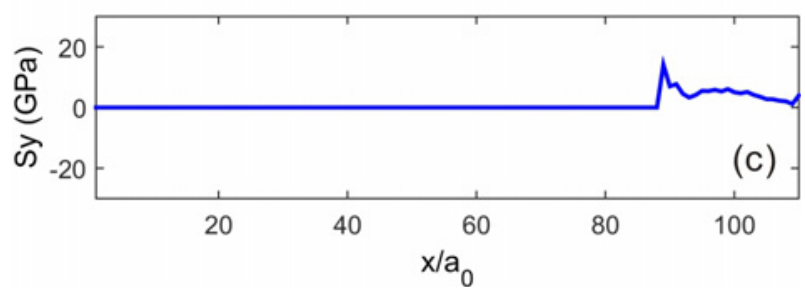

Fig. 5. Visualization of the first dislocation emission on the inclined slip systems $\langle 111\rangle\{112\}$ at the edge crack $a / W=0.8$ in the middle of the crystal, time step $11600\left(K_{\mathrm{A}}(t)=1.16 K_{\mathrm{G}}\right) v_{\mathrm{A}}(t)=8.53$ : (a) by means of atomic coordinates; (b) by means of local kinetic energies of individual atoms: the value 8 on the color scale corresponds to a maximum of the local kinetic energy in the crystal; at the bright dislocation cores $E_{\text {kin }}(l i)=0.33 \times 10^{-20} \mathrm{~J}$, while the average value per one atom in the crystal is $E K I N / N P O I N=0.10 \times 10^{-22} \mathrm{~J} ;$ (c) decrease of the stress concentration at the crack tip after dislocation emission.

Maxwell-Boltzmann relations for 3 degrees of freedom, the kinetic energy at the dislocation core corresponds to the temperature of about $159 \mathrm{~K}$, while the average temperature in the atomistic sample (Fig. 1) is $4.8 \mathrm{~K}$.

The motion of the individual dislocation segments may differ in 3D. Here we follow and evaluate the generation and kinetics of motion of the lower edge dislocation lying below crack faces in atomistic Figs. 5a, $6 \mathrm{a}$, and $7 \mathrm{a}$, which is the right dislocation in Figs. 5b, $6 \mathrm{~b}$, and $7 \mathrm{~b}$, by the map of the local atomic kinetic energies. In this case, Burgers vector lies in the observation plane (110) and the direction [110] of the dislocation line is parallel with crack front and perpendicular to the observation plane - see the precise notation in Figs. 5a, 7b.

The dislocations in Fig. 5a at time step 11600 $\left(K_{\mathrm{A}}(t)=1.16 K_{\mathrm{G}}\right)$ begin to move away from the crack tip, and the right free surface does not still influence the AE signal significantly in Fig. 5b. The character of the AE signal in Fig. 5b is predominantly given by the 


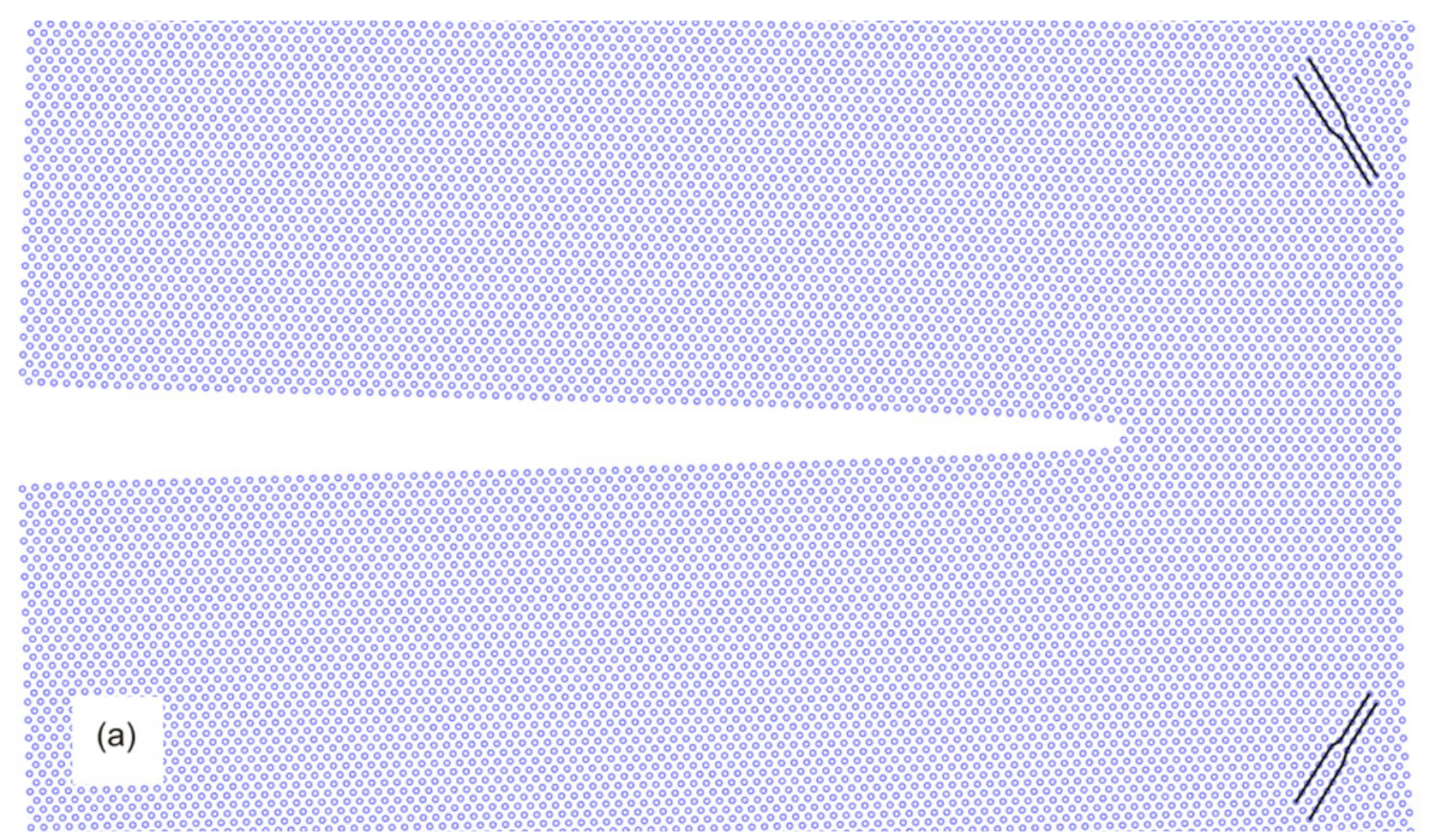

$$
\left(E_{\text {kin }}\left(I_{i}\right) / 10^{-22}\right)^{1 / 2} \text {, double layer=8 }
$$
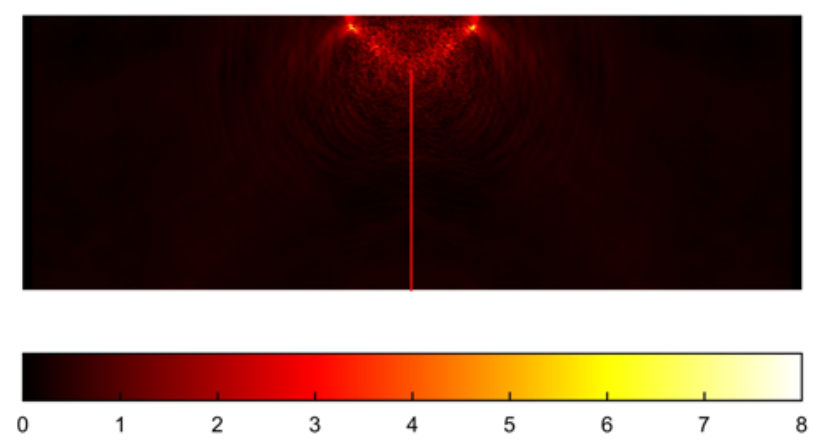

(b)

Fig. 6. The motion of the dislocations away from the crack front in the middle of the crystal, time step $11800\left(K_{\mathrm{A}}(t)=\right.$ $1.18 K_{\mathrm{G}}$ ): (a) dislocation position using atomic coordinates; (b) using the local kinetic energies, representing the source of AE signal.

envelope of the shear waves emitted from the moving dislocation cores in the two active slip systems $\langle 111\rangle$ $\{112\}$, similar to [16].

A different character of AE signal from the moving dislocations approaching the free surface is shown in Fig. $6 \mathrm{~b}$ at time step $11800\left(K_{\mathrm{A}}(t)=1.18 K_{\mathrm{G}}\right)$. In this case, the AE patterns are already strongly influenced by the near free surface. Dislocation cores in Fig. 6b are visible using the yellow "hot" points. At this time step, the atomic position of the lower dislocation in Fig. 6a corresponds to $r=35 b$ (measured from the lower free crack face).

Figure 7 shows the situation at time step 11885 when the dislocations penetrate the right free surface layers. One may observe that the atomic configuration in Fig. 7a does not well define the position of the dislocation core, as the core is being split and looks like a collective motion of atoms, which creates a surface step. In comparison with Fig. 6b, the envelope of the shear waves emitted in Fig. 7b is more separated, via the dark region (with low kinetic energy), from the entire wave region generated during the previous dislocation motion. This indicates an acceleration of the dislocations close to the free surface.

The atomic configurations are prior to vanishing of the dislocations at the free surface and, after that, 


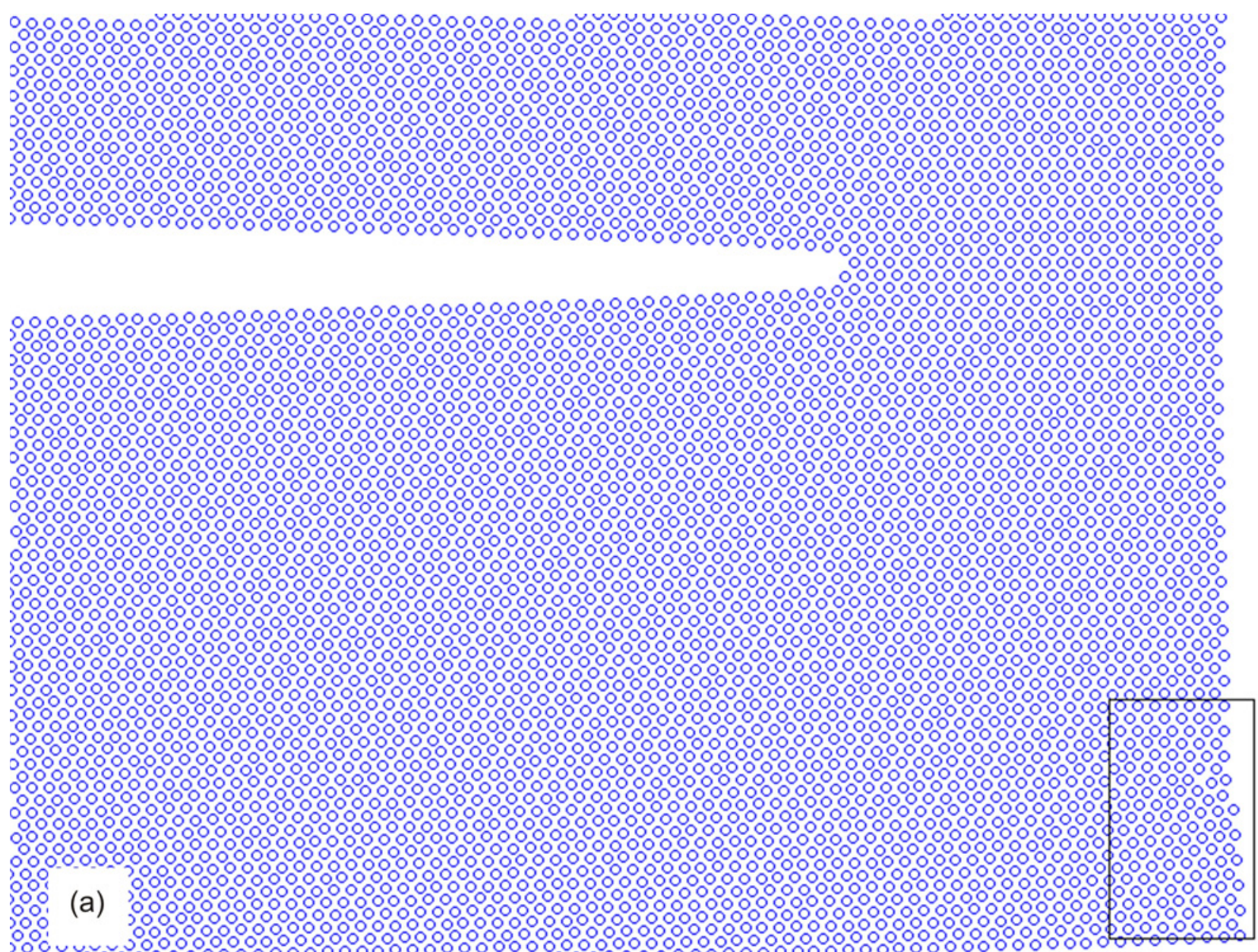

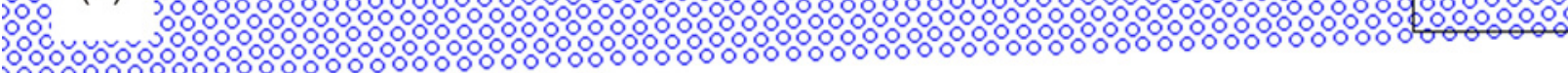

$$
\left(\mathrm{E}_{\mathrm{kin}}\left(\mathrm{I}_{\mathrm{i}}\right) / 10^{-22}\right)^{1 / 2} \text {, double layer=8 }
$$

$[\overline{1} 11](\overline{1} 1 \overline{2})$
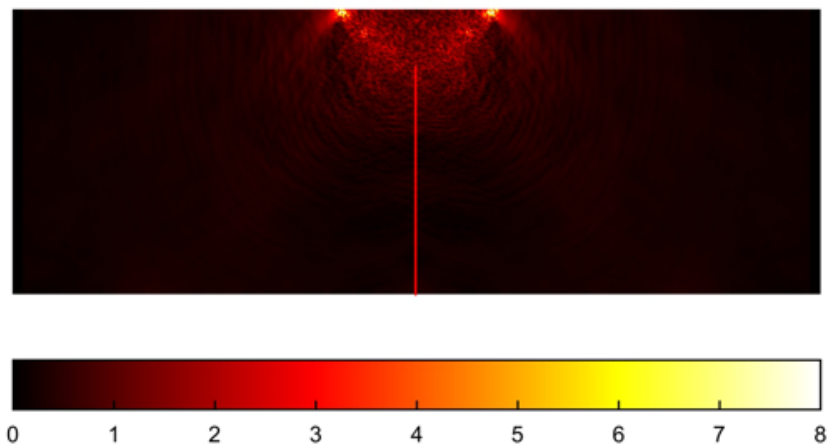

(b)

Fig. 7. The arrival of the emitted dislocations to the free sample surface, middle of the crystal, time step 11 885: (a) the complex (lighted) atomic configuration before the arrival of the dislocation to the free surface; (b) acoustic emission signal from the transonic dislocation motion using the local kinetic energies.

are presented in Fig. 8. At time step 11880 (Fig. 8a) one can localize the position of the core to $r=42-$ 43b, while at time steps 11890 (Fig. 8b) and 11900 (Fig. 8c), one may already observe the surface steps at their final position $r=46 b$, as denoted by the arrow. Monitoring of the dislocation motion is shown in Fig. 9 and Table 1 using the dimensionless positions $r / b$ of the lower dislocation in dependence on dimen- 

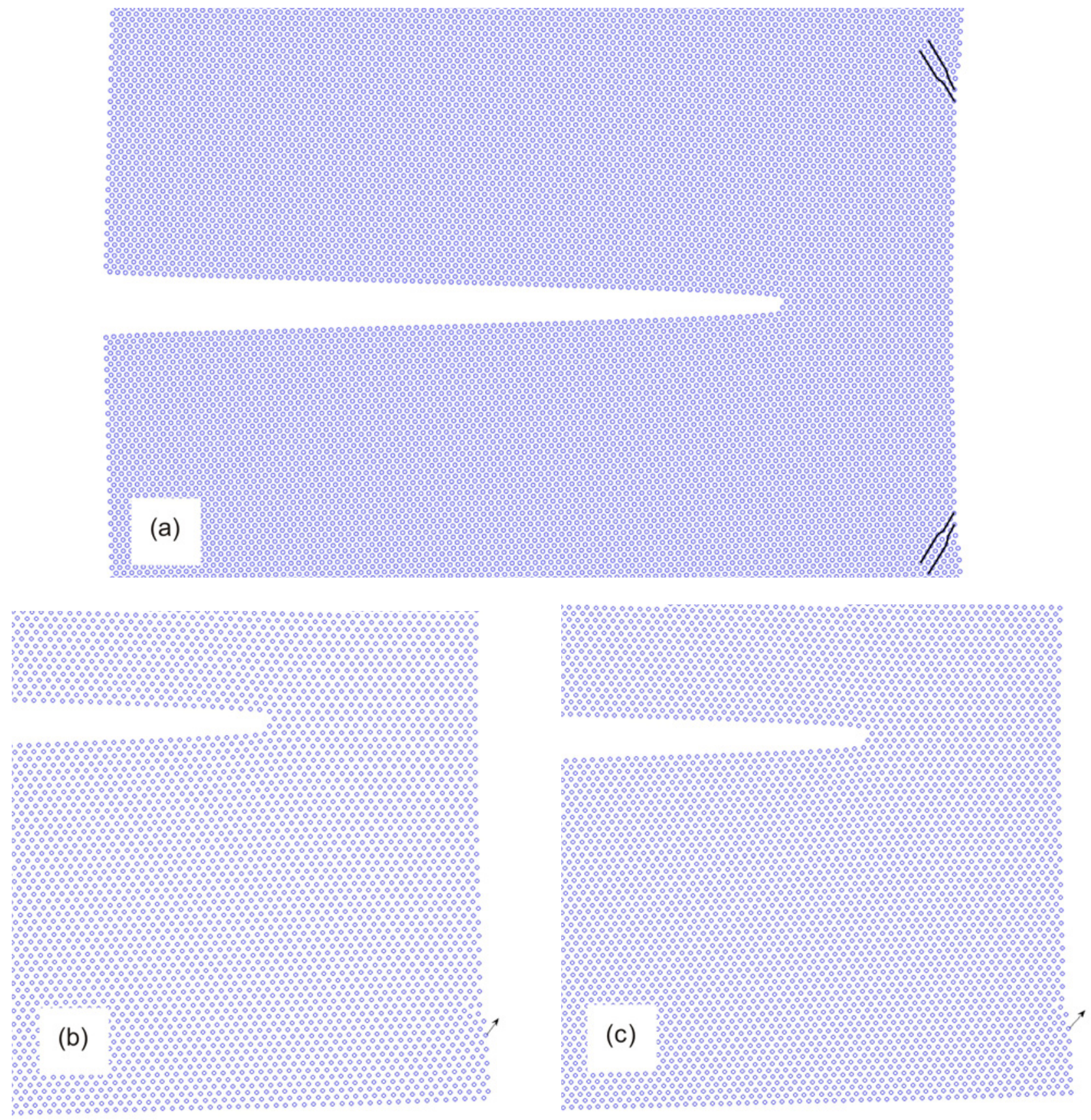

Fig. 8. (a) Dislocation position at time step $11880\left(K_{\mathrm{A}}(t)=1.188 K_{\mathrm{G}}\right)$; (b) surface steps at time step $11890\left(K_{\mathrm{A}}(t)=\right.$ $\left.1.189 K_{\mathrm{G}}\right)$; (c) time step $11900\left(K_{\mathrm{A}}(t)=1.190 K_{\mathrm{G}}\right)$ denoted by the arrow.

sionless time $\Delta t / \mathrm{h}$, where $\Delta t=t-11600 h$. The line corresponds to an average subsonic dislocation velocity of $V_{\text {disl }}=2210 \mathrm{~m} \mathrm{~s}^{-1} \pm 407 \mathrm{~m} \mathrm{~s}^{-1}$ that was obtained by the least-square method in the time interval $<11600 h, 11880 h>$, or according to Fig. 9 in the interval $\Delta t / \mathrm{h} \in<0,280\rangle$.

In the time interval $<11880 h, 11900 h>$, i.e., close to the free surface, the slope of the curve differs, as may be seen in Fig. 9 for $\Delta t / \mathrm{h} \in<280,300>$. The dislocation in MD accelerates at a very close distance of about $4 b$ from the right free surface. It leads to an in- stantaneous dislocation velocity in the $\langle 111\rangle$ direction, either transonic $V_{\text {disl }}=(46-43) b / 20 h=3724 \mathrm{~m} \mathrm{~s}^{-1}$ above $C_{\mathrm{T}}=3007 \mathrm{~m} \mathrm{~s}^{-1}$ [10], or to supersonic $V_{\mathrm{disl}}=$ $(46-43) b / 10 h=7447 \mathrm{~m} \mathrm{~s}^{-1}$ above $C_{\mathrm{L}}=6487 \mathrm{~m} \mathrm{~s}^{-1}$ [10], if we suppose that the surface step was already created at the time step 11890 .

In Fig. 10 we present a simplified reconstruction of the acoustic emission from the transonic dislocation motion nearby the free surface in the framework of an anisotropic continuum of our crystal symmetry. For the potential used, the velocity of the transver- 
Table 1. Global energy and force balance for the first dislocation emission from the edge crack $a / W=0.8$ in mode I under constant displacements conditions, loading rate $14.7 \AA / 20000 h(0.010 \mathrm{~K} \mathrm{G} / \mathrm{ps})$. The energies are in units of $10^{-15} \mathrm{~J}$, $E=E P O T+E K I N$ and the forces $F_{\text {disl }}$ are in units of $\mathrm{N} \mathrm{m}^{-1}$. Time sampling $\Delta t / \mathrm{h}$ and the positions $r / b$ of the lower emitted dislocation correspond to Fig. 9.

\begin{tabular}{|c|c|c|c|c|c|c|c|}
\hline time step $n$ & EPOT & $E K I N$ & $E$ & $r / b$ & $\Delta r / b$ & $F_{\text {disl }}$ & $\Delta t / \mathrm{h}$ \\
\hline 11600 & 0.2446 & 0.0150 & 0.2596 & 18 & 0 & 0 & 0 \\
\hline 11620 & 0.2477 & 0.0158 & 0.2635 & 20 & 2 & -0.033 & 20 \\
\hline 11640 & 0.2444 & 0.0171 & 0.2615 & 22 & 4 & 0.033 & 40 \\
\hline 11660 & 0.2445 & 0.0181 & 0.2626 & 24 & 6 & 0.011 & 60 \\
\hline 11680 & 0.2440 & 0.0195 & 0.2635 & $25-26$ & $7-8$ & 0.053 & 80 \\
\hline 11700 & 0.2439 & 0.0205 & 0.2644 & $27-28$ & $9-10$ & 0.049 & 100 \\
\hline 11720 & 0.2433 & 0.0221 & 0.2654 & $28-29$ & $11-12$ & 0.075 & 120 \\
\hline 11740 & 0.2428 & 0.0236 & 0.2664 & $30-31$ & $12-13$ & 0.095 & 140 \\
\hline 11760 & 0.2423 & 0.0252 & 0.2675 & 32 & 14 & 0.108 & 160 \\
\hline 11780 & 0.2416 & 0.0268 & 0.2684 & 34 & 16 & 0.124 & 180 \\
\hline 11800 & 0.2406 & 0.0289 & 0.2695 & $35-36$ & 18 & 0.147 & 200 \\
\hline 11820 & 0.2395 & 0.0309 & 0.2704 & 37 & 19 & 0.177 & 220 \\
\hline 11840 & 0.2386 & 0.0328 & 0.2714 & 39 & 21 & 0.189 & 240 \\
\hline 11860 & 0.2376 & 0.0348 & 0.2724 & 41 & 23 & 0.201 & 260 \\
\hline 11880 & 0.2357 & 0.0376 & 0.2733 & $42-43$ & $24-25$ & 0.240 & 280 \\
\hline 11900 & 0.2312 & 0.0433 & 0.2745 & 46 & 28 & 0.316 & 300 \\
\hline
\end{tabular}

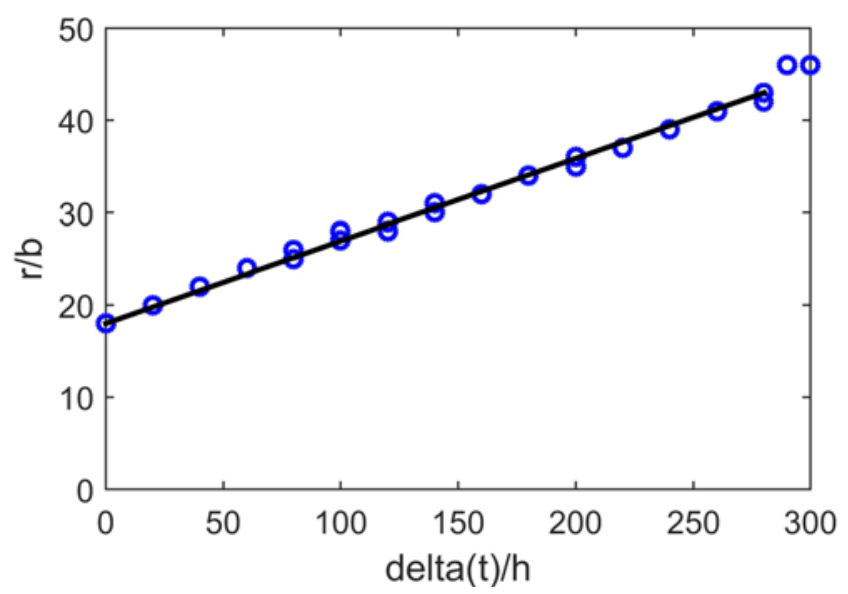

Fig. 9. Dislocation motion by means of dimensionless position $r / b$ from crack faces in dependence on dimensionless time $\Delta t / \mathrm{h}$, where $\Delta t=t-11600 \mathrm{~h}$. The line corresponds to an average dislocation velocity $V_{\text {disl }}=$ $2210 \mathrm{~m} \mathrm{~s}^{-1} \pm 407 \mathrm{~m} \mathrm{~s}^{-1}$. The interval $\Delta t / \mathrm{h}=<0,300>$ corresponds to applied stress intensity $K_{\mathrm{A}}(t)=<1.16 K_{\mathrm{G}^{-}}$ $1.19 K_{\mathrm{G}}>$.

sal (shear) waves in the $\langle 111\rangle$ direction corresponds to $C_{\mathrm{T}}=3007 \mathrm{~m} \mathrm{~s}^{-1}$, and for the longitudinal waves, it is $C_{\mathrm{L}}=6487 \mathrm{~m} \mathrm{~s}^{-1}$ [10], as mentioned above. In an anisotropic medium, only some crystallographic directions (in our case $\langle 100\rangle,\langle 011\rangle$ and $\langle 111\rangle$ ) transfer pure longitudinal or pure transversal mode, in other directions the elastic waves propagate as a quasilongitudinal (qL) or quasi-transversal (qT) waves [16]. Note that in [16] we treated dislocation emission from a short central crack in an extended crystal and the influence of free surfaces was not examined in [16]. Figure 10a shows the section of the ray (wave) surfaces using the group velocities of the quasi-longitudinal $\mathrm{qL}$ and quasi-transversal qT (shear) waves in our observation plane (110) in the bcc iron crystal under plane strain conditions. The point source of the anisotropic AE signal lies in the middle of Fig. 10a and may represent, e.g., the crack tip point or dislocation core. The axis of the potential crack extension in Fig. 10 and Figs. 5b, 6b, and 7b corresponds to [001] direction, see Fig. 1. Our slip system $\langle 111\rangle\{112\}$ for dislocation emission is oriented under an angle of about $55^{\circ}$ ( $\tan \alpha=\sqrt{2})$ concerning the [001] direction of the potential crack growth as mentioned above. The dislocation emission from the crack tip is a shear process, and so it can be modeled as a moving point source of the shear qT waves along the slip direction $\langle 111\rangle$ inclined under $\sim 55^{\circ}$ concerning [001] direction - see Fig. 10b, where the moving point source is denoted via $(+)$ for a dislocation velocity of about $1.36 C_{\mathrm{T}}$. The crosses in the $\langle 111\rangle$ direction correspond to Burgers vector. The envelope of the shear qT waves emitted in Fig. 10b represents an AE signal arising from the transonic motion of the right (lower) emitted dislocation in an extended (unbounded) observation plane (110), i.e., it shows the situation during dislocation motion in the interval from the position $42 b$ up to $46 b$, unimpeded by the free surface and by the back wave reflections from free surface. If we perform in Fig. 10b a cut selection nearby the last position (46b) of the dislocation core $(\bullet)$ to model the arrival of the dislocation close to the free surface, we obtain in Fig.10c an AE signal similar to Fig. 10d coming from the transonic motion of the right dislocation approaching the free (001) sur- 

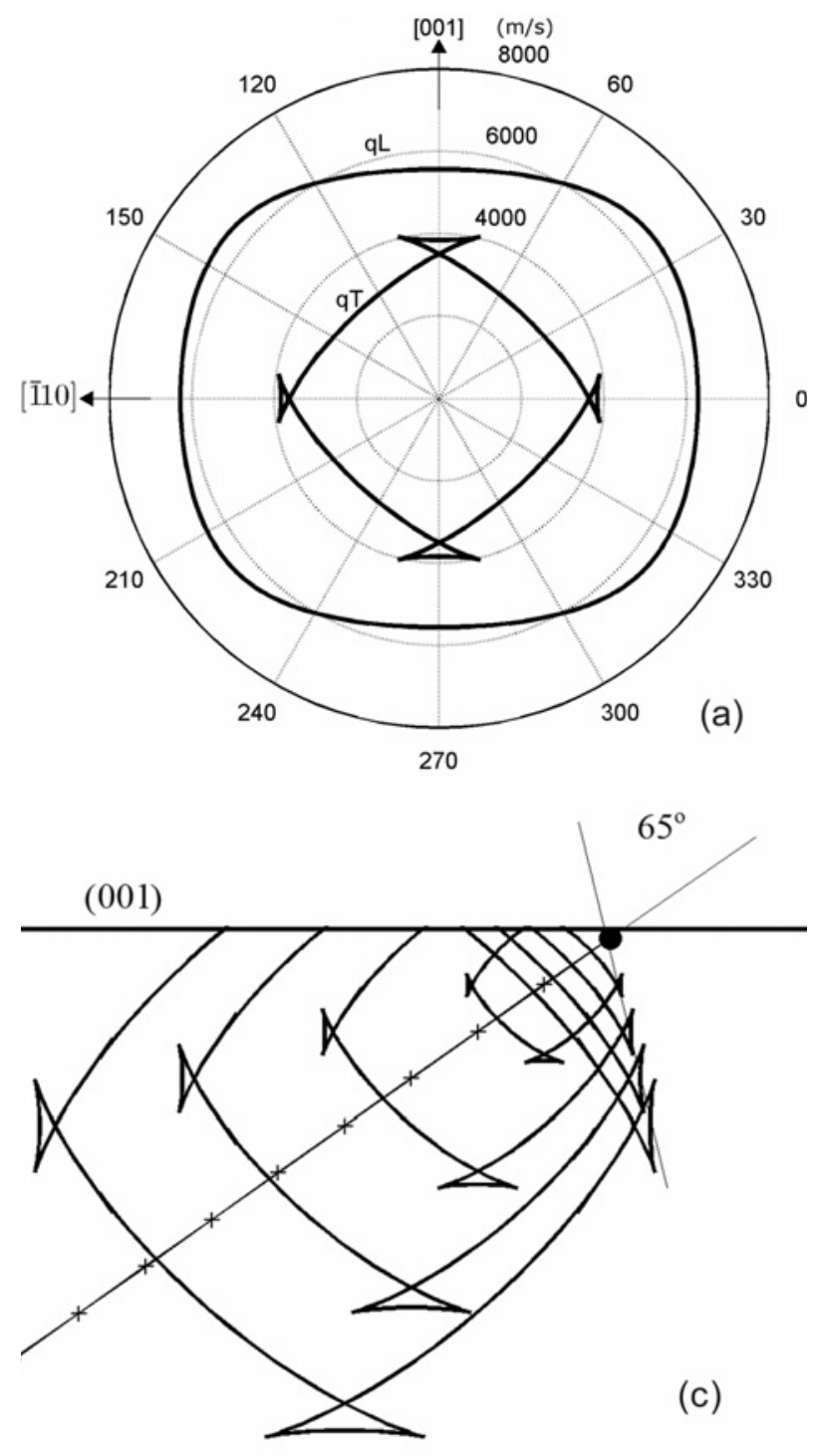
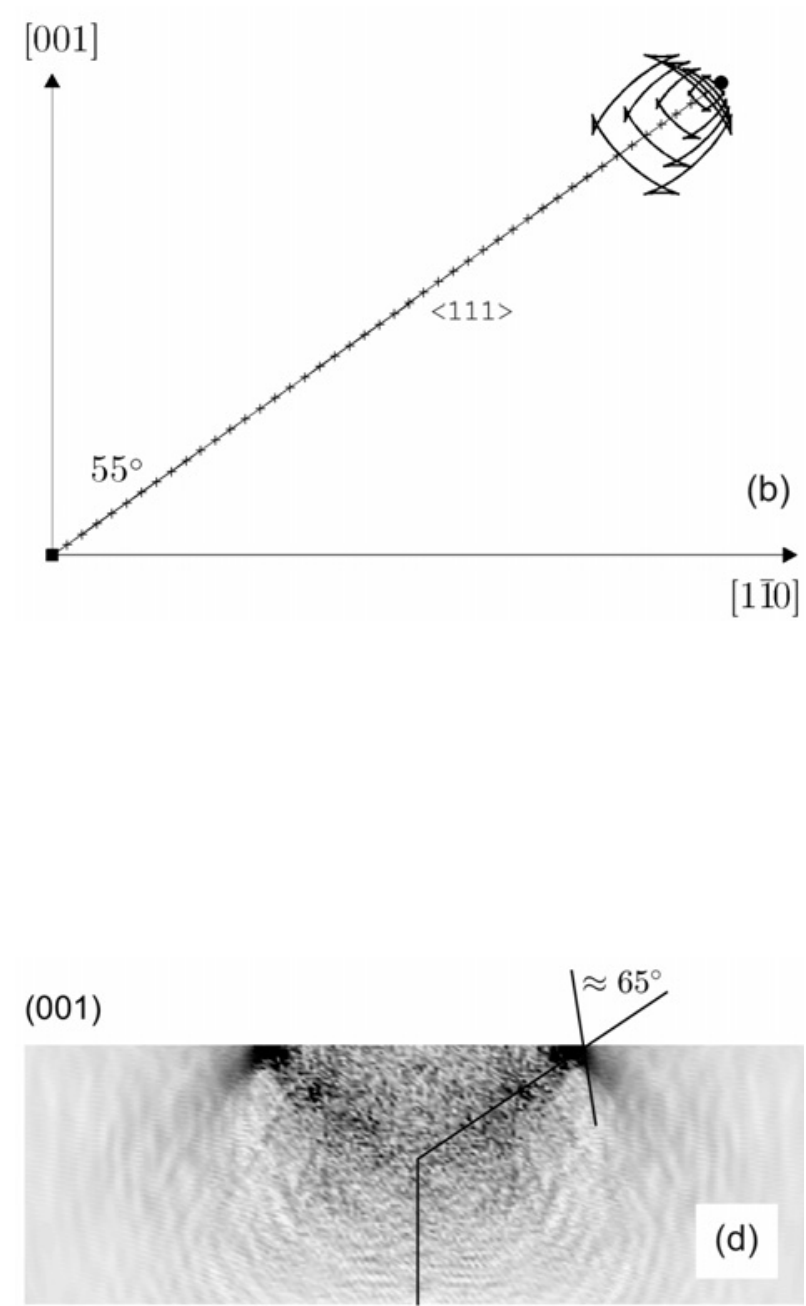

Fig. 10. Reconstruction of the acoustic emission (AE) sources in anisotropic continuum of our crystal symmetry: (a) section of the ray (wave) surfaces: group velocities of the quasi-longitudinal (qL) and quasi-transversal (qT) on (110) plane of bcc iron under plane strain; (b) AE patterns nearby the final position $(\bullet)$ of the dislocation core, generated by the transonic dislocation, modeled as a moving source $(+)$ of the qT-waves with a velocity $V_{\text {disl }}=1.36 C_{\mathrm{T}}$. Initial position of the dislocation is identical with the crack tip point denoted via $(\mathbf{\square})$ and the moving positions $(+)$ correspond to Burgers vector in the slip direction $\langle 111\rangle$ inclined under an angle of about $55^{\circ}$ with respect to axis of the potential crack extension in the direction [001]; (c) simplified reconstruction at the free surface created via a cut selection from Fig.10b nearby the final position of the right dislocation core $(\bullet)$ approaching the free surface and Mach angle of about $65^{\circ}$ corresponding to transonic velocity $1.36 C_{\mathrm{T}} ;$ (d) comparison with a detail from MD for the transonic dislocation approaching the free (001) surface in Fig. 7b (gray color scale 3 in Matlab).

face in MD at time step 11885 (Fig. 10d presents a detail from MD results in Fig. 7b, performed with a gray color scale 3 in MATLAB).

The simplified reconstruction does not include the back wave reflections acting in $\mathrm{MD}$ and the previous radiation coming from dislocation generation and subsequent subsonic dislocation motion. Nevertheless, the shape of the AE signal and Mach angle of about $65^{\circ}$ following from the wave reconstruction in Fig. 10c roughly describe the independent MD signal in Fig. 10d at the free (001) surface. It implies that the strongest AE signal from the crack-induced dislocation emission is transferred via $\mathrm{qT}$ waves, which is useful information for the experimental AE detection with the velocity sensitive transducers.

Note that the definition (classification) of the fast defects in an anisotropic medium differs in various publications since the wave propagation in anisotropic crystals is more complex in comparison with the isotropic continuum, where just two velocities of the 
longitudinal $\left(C_{\mathrm{L}}\right)$ and transversal $\left(C_{\mathrm{T}}\right)$ waves can exist. An analytical solution by Eshelby [6] for the displacement and stress field of a uniformly moving edge dislocation in isotropic continuum discovers a special case when the dislocation moves with a transonic speed $V_{\text {disl }}=\sqrt{2} C_{\mathrm{T}}$. In this case, the normal component of the stress field generated by the moving edge dislocation vanishes (i.e., the dislocation does not radiate the stress waves in the direction perpendicular to slip plane) and it may exist without external forces applied in the slip direction (shear mode II). This state is called "radiation free" in later studies, e.g. [11].

The qT waves are often denoted as the quasi-shear (qS) waves. It is well known that the slow $\left(\mathrm{qS}_{1}\right)$ and faster quasi-transversal qT waves $\left(\mathrm{qS}_{2}\right)$ must be recognized in anisotropic cubic crystals. For example, the velocity of the faster qT waves (presented in Fig. 10) in the $\langle 110\rangle$ directions is given by the elastic constant $C_{44}$, while the velocity of the slower shear wave $\left(\mathrm{qS}_{1}\right)$ in these directions is given by $\left(C_{11}-C_{12}\right) / 2$. The waves have different polarization. The faster qT wave is polarized in the same plane where the direction of propagation lies, while the slower $\mathrm{qT}\left(\mathrm{qS}_{1}\right)$ wave is polarized in a perpendicular plane.

In our crystal orientation (Fig. 1) the faster qT $\left(\mathrm{qS}_{2}\right)$ wave propagating in the $[\overline{1} 10]$ direction is polarized in the [001] direction, while the slower transversal (shear) wave $\left(\mathrm{qS}_{1}\right)$ is polarized in the perpendicular [110] direction. In Fig. 10 we do not consider the slower transversal wave $\mathrm{qS}_{1}$ since we use plane strain conditions with zero displacements in the perpendicular [110] direction. These are relevant for the edge dislocations emitted from the crack in the slip systems $\langle 111\rangle\{112\}$ since the Burgers vector lies on the observation plane (110) (there is no component in the perpendicular direction [110]) - see, e.g., Figs. 4, 5a.

The studies $[11,18]$ are based on Eshelby's work $[6,17]$ and they introduced following definitions in anisotropic medium: the regime with $V_{\text {disl }}<C\left(\mathrm{qS}_{1}\right)$ is called subsonic (it does not occur in this study), the interval $C\left(\mathrm{qS}_{1}\right)<V_{\text {disl }}<C\left(\mathrm{qS}_{2}\right)$ is named as the first transonic regime (it is called subsonic in this study), the interval $C\left(\mathrm{qS}_{2}\right)<V_{\text {disl }}<C(\mathrm{qL})$ is the second transonic regime (here we say simply transonic) and $V_{\text {disl }}>C(\mathrm{qL})$ is called supersonic, as here.

It is interesting to note that the study [18] presents the character of acoustic emission by Huygens type geometric construction of Mach cones from analytical solutions for moving dislocations but for the different slip system $\langle 111\rangle\{1 \overline{1} 0\}$ in bcc iron. Here, two Mach cones are visible on a $\{11 \overline{2}\}$ plane, caused by the $\mathrm{qS}_{1}$ and $\mathrm{qS}_{2}$ waves. This type of slip system is theoretically available for our crack orientation but was not activated in this study because of the lower Schmid factor (0.41) in comparison with our active $\langle 111\rangle\{112\}$ slip systems (Schmid factor 0.47). However, we monitored the activity of the oblique slip systems of the type $\langle 111\rangle\{1 \overline{1} 0\}$. at the crack $(\overline{1} 10)[110]$ in MD simulations under mode I at room temperature.

The next part of the discussion is devoted to possible sources for dislocation acceleration observed in MD. Figure 5c illustrates that the stress concentration induced by the crack (see Fig. 2) almost disappears at time step 11600 since it is spent by the dislocation emission process and so other sources should be considered to explain the acceleration of dislocations toward the free surface. This is discussed below.

By continuum theory of dislocations, the strain energy $U$ accumulated around a dislocation depends on its position $\boldsymbol{r}$ in a finite body. The interaction dislocation-free surface can be described generally by a self-force $\boldsymbol{F}=-\mathrm{d} U / \mathrm{d} \boldsymbol{r}$. Acceleration of dislocations in the direction $\boldsymbol{r}$ parallel with $\boldsymbol{b}$ for the discrete time and position sampling in Table 1 can be approximated by the Newton's equation of motion for unit length of the dislocation line:

$$
\begin{gathered}
M_{1} \Delta V_{\mathrm{disl}} \Delta t=F+b\left(\tau_{\mathrm{s}}-\tau_{\mathrm{PN}}\right), \\
F=-\Delta U / \Delta r
\end{gathered}
$$

where $M_{1}=m / a_{0} \sqrt{2}=2.2877 \times 10^{-16} \mathrm{~kg} \mathrm{~m}^{-1}$ and $m=9.274012 \times 10^{-26} \mathrm{~kg}$ is the mass of one iron atom, $\tau_{\mathrm{s}}$ is the nominal shear stress from external loading, $\tau_{\mathrm{s}}=\sigma_{0} \sin \alpha \cos \alpha$ (in our case $\alpha \sim 55^{\circ}$ and the Schmid factor is $\sin \alpha \cos \alpha=0.47)$, the corresponding slip force is $F_{\mathrm{s}}=\tau_{\mathrm{s}} b$, further $\tau_{\mathrm{PN}}$ is the lattice resistance against motion of an edge dislocation given by Peierls-Nabarro model as $\tau_{\mathrm{PN}}=\{2 \mu /(1-$ $\nu)\} \exp \{-2 \pi d / b(1-\nu)\}$ and $F_{\mathrm{PN}}=\tau_{\mathrm{PN}} b$ is the $\mathrm{PN}$ force acting on unit length of dislocation line. Replacing shear modulus $G$ by the anisotropic elastic constant $\mu=\left(C_{11}-C_{12}+C_{44}\right) / 3=0.713 \times 10^{11} \mathrm{~N} \mathrm{~m}^{-2}$ (relevant for the slip system $\langle 111\rangle\{112\}$ ), further Poisson's ratio via the relation $\nu=0.596$ valid [15] for our crystal symmetry and the potential in use $[5,6]$ and using for our slip system $\langle 111\rangle\{112\}$ the interplanar distance $d=a_{0} / \sqrt{6}=1.1702 \AA$ and Burgers vector $b=2.4825 \AA$ we obtain an estimate of PN stress $\tau_{\mathrm{PN}}=$ $232 \mathrm{MPa}$. The estimate is comparable with the atomistic results $\tau_{\mathrm{PN}} \sim 3 \times 10^{-3} \mu$ presented in [19], [26] for the edge dislocations $\langle 111\rangle\{112\}$ in bcc crystals. Corresponding $\mathrm{PN}$ force is $F_{\mathrm{PN}}=\tau_{\mathrm{PN}} b=0.057 \mathrm{~N} \mathrm{~m}^{-1}$. In the time interval $<11600 h, 11900 h>$ monitored in $\mathrm{MD}$, the nominal tension stress from external loading varies only slightly from $\sigma_{0}(11600)=4.28 \mathrm{GPa}$ up to $\sigma_{0}(11900)=4.39 \mathrm{GPa}$. The slip force acting per unit length of the dislocation line is also almost constant: $F_{\mathrm{s}}(11600)=0.4994 \mathrm{~N} \mathrm{~m}^{-1}$ while $F_{\mathrm{s}}(11900)=$ $0.5122 \mathrm{~N} \mathrm{~m}^{-1}$.

It is interesting to note that PN stress (needed for dislocation motion) is approximately by 3 orders smaller in comparison with the critical shear stress needed for dislocation generation from the crack tip. 
In our case the stress barrier for dislocation generation in the $\langle 111\rangle\{112\}$ slip system is $\tau_{\mathrm{c}}=16.3 \mathrm{GPa}$, as follows from block-like shear simulations in perfect bcc iron crystals with the used potential - see $[15$, 16]. The value of $\tau_{\mathrm{c}}$ is comparable with the estimate of the ideal shear strength $\mu / 2 \pi$ in perfect crystals by Frenkel [3], which is included in the models [14, 15] for crack induced dislocation generation, together with PN stress. This means that the level of the critical stress intensity factor $K_{\text {disl }}$ is given predominantly by the high-stress barrier for dislocation generation that is supplied by the high-stress concentration at the crack tip (see Fig. 2).

Koehler [1] proposed a simplified isotropic model for the calculation of an image force $F_{\mathrm{R}}$ that drives out the dislocation from the body to decrease the strain energy in the system. A fictitious mirror dislocation of the opposite sign is introduced. The dislocation lies outside the crystal in the same slip plane at the same distance $R$ from the free surface. The interaction force $F_{\mathrm{R}}$ between the real and mirror dislocation is attractive, called the image or mirror force and it drives out the real dislocation from the crystal. The model preserves the conditions of the free surface in the normal direction, but not in the tangential (shear) direction. (It is easy to show in polar coordinates $r, v$ that $\sigma_{r r}=$ 0 and $\sigma_{r v} \neq 0$ for $\left.v=0\right)$. In the case of the edge dislocations in isotropic continuum, the image force is $F_{\mathrm{R}}=\left\{G b^{2} / 2 \pi(1-v)\right\} / 2 R=$ const $/ R$. Replacing $G$ and Poisson's ratio by the anisotropic values $\mu$ and $v$ given above, we obtain const $=0.8655 \times 10^{-9} \mathrm{~N}$ for our case. At time step 11600 the distance from the free sample surface in MD is $R=(46-18) b=28 b$ and $F_{\mathrm{R}}=0.125 \mathrm{~N} \mathrm{~m}^{-1}$. At the time step 11800 the distance $R=(46-36) b$ and $F_{\mathrm{R}}=0.349 \mathrm{~N} \mathrm{~m}^{-1}$.

The image forces are of a long-range, given approximately by the relation $F_{\mathrm{R}}=F_{\mathrm{PN}}$, which is in our case by about $60 b$, i.e., about 30 lattice parameters from the free surface (001). The acceleration of the emitted dislocations toward the right free surface caused by the mirror forces themselves can be approximated by the Newton's equation of motion using $F_{\mathrm{R}}$ instead of $F$ and neglecting the second term. Using the static image forces $F_{\mathrm{R}}$, the dislocation accelerates already from time step 11620 (in contradiction with MD results in Fig. 9) and penetrates the free surface in transonic regime much earlier in comparison with $\mathrm{MD}$.

Better agreement with MD results could be obtained using the elasto-dynamic model [2] for the image forces. The study brings an important improvement, so-called retardation time. The injected (emitted) dislocation does not feel the image force until the stress wave emitted by the complete dislocation arrives at the free surface (now, the surface feels that there is some dislocation in the bulk of the crystal). After that, the stress waves are reflecting the dislocation (and now, the injected dislocation knows that there is some free surface). This occurs when their dimensionless parameter $t / a l=2$. Up to this moment, the dynamic image force is zero. Here the time $t$ is measured from the time of the injection and the parameter $a l=R / C_{\mathrm{L}}$ in our case.

The paper also introduces a dynamic correction $f(t)$ (Fig. 5 in [2]) for image forces that a uniformly moving edge dislocation feels. The dynamic image force is introduced as $F_{\mathrm{dyn}}=2 f(t) F_{\mathrm{R}}$. The isotropic function $f(t)$ from [2] can be modified to our anisotropic case by using our anisotropic ratio $C_{\mathrm{T}} / C_{\mathrm{L}}=0.4635$ and the parameter $V_{\text {disl }} / C_{\mathrm{T}}=0.735$. Here $C_{\mathrm{L}}$ and $C_{\mathrm{T}}$ mean the velocities of the longitudinal and transversal (shear) waves in the $\langle 111\rangle$ slip direction. The dynamic correction $f(t)$ in our case is similar to the isotropic case shown in Fig. 5 in [2] concerning the parameters $C_{\mathrm{T}} / C_{\mathrm{L}}=0.5$ and $V_{\mathrm{disl}} / C_{\mathrm{T}}=$ 0.8 .

Assuming that the time of the injection of the complete edge dislocation corresponds to $11600 \mathrm{~h}$ in agreement with $\mathrm{MD}$ data, then their parameter $R / C_{\mathrm{L}}=$ $28 b / C_{\mathrm{L}}=107 \mathrm{~h}$ in our case and $t=\Delta t$ is related to the time step 11600 as in Fig. 9, then up to time step 11814 (when $\Delta t /\left(R / C_{\mathrm{L}}\right)=2$ ) the dynamic image force $F_{\text {dyn }}$ is zero, and so, no acceleration of the edge dislocation is expected before time step 11820 which better complies with MD data.

Nevertheless, the retardation time in MD differs somewhat from the model in [2]. The first part of retardation is the same: the time interval needed for the arrival of the longitudinal emitted waves to the right free sample surface is $\Delta t_{0}=28 b / C_{\mathrm{L}}=107 \mathrm{~h}$. Unlike the model [2] in our case, the emitted (injected) dislocation immediately moves with a velocity $V$ and meets the reflected L-wave at a time $\Delta t_{1}$ when its position from the surface $R=R_{0}-V \Delta t_{0}-V \Delta t_{1}=C_{\mathrm{L}} \Delta t_{1}$. Since $R_{0}=C_{\mathrm{L}} \Delta t_{0}$, the unknown time $\Delta t_{1}$ by the previous equation is $\Delta t_{1}=\Delta t_{0}\left(C_{\mathrm{L}}-V\right) /\left(C_{\mathrm{L}}+V\right)$. Thus, the total time of retardation $\Delta t=\Delta t_{0}+\Delta t_{1}=$ $\Delta t_{0} 2 C_{\mathrm{L}} /\left(C_{\mathrm{L}}+V\right)$ and the dimensionless parameter in our case is $\Delta t /\left(R_{0} / C_{\mathrm{L}}\right)=2 C_{\mathrm{L}} /\left(C_{\mathrm{L}}+V\right)$.

A similar relation can be derived for the transversal (shear) waves $C_{\mathrm{T}}$. For $V=2210 \mathrm{~m} \mathrm{~s}^{-1}$ from MD it leads to a different limiting value of 1.4953 instead of 2 , which is valid only for $V=0$. This means that, for the treatment of MD results, we should consider $F_{\text {dyn }} \neq 0$ as already behind the time step 11760 which again causes an early acceleration of the dislocation in comparison with the MD results in Fig. 9.

If we also apply the procedure to the left crack free face, where the mirror dislocation of opposite sign $\left(F_{\mathrm{r}}<0\right)$ should hinder the motion of dislocation to the right surface, we find that the resulting $F_{\text {dyn }}=$ $f(t)\left(F_{\mathrm{R}}+F_{\mathrm{r}}\right) \neq 0$ can act from the time step 11780, leading again to the early acceleration with respect to MD results since also $F_{\text {dyn }} \sim 1 / R$. Such dependence is too strong, e.g., in nanocrystals, the model could 
cause a dislocation-free state, as pointed out in [24].

Another reason why the improved elasto-dynamic model [2] of the image forces in the isotropic continuum is inadequate for treatment of our MD results, is the anisotropy. The angular distribution of the static stress field differs in bcc lattice [1c] in comparison with isotropic continuum while the radial dependence $\sim 1 / R$ is preserved. The propagation of the stress waves in anisotropic continuum differs as well from the isotropic case, where the $\mathrm{L}$ and $\mathrm{T}$ waves have a circular character. This is demonstrated in Fig. 10a.

This means that the dynamic correction functions $f(t)$ in the anisotropic medium may differ significantly from the analytical form presented in [2]. Moreover, finally, at the free surfaces, the limiting velocity in an isotropic medium is the Rayleigh velocity of surface waves $C_{\mathrm{SW}}<C_{\mathrm{T}}$, which need not be valid in anisotropic medium (see [27] and the discussion below).

As mentioned in the Introduction, another possible source for acceleration of dislocations at the right free surface in MD is surface stress. The right free surface is relaxed (the surface relaxation was performed before loading, mentioned in Sec. 2), which slightly changes atomic positions at the free surface and leads to surface stress. As mentioned in the Introduction, the surface relaxation (and consequently surface stress) is a real phenomenon in MD as well as in experiments.

As to the experimental data, the experiments on surface relaxation in bcc iron crystals were performed [20] at room temperature. Here normal (N) and tangential ( $\mathrm{T}$ shear) relaxation of individual surface planes in several crystallographic directions were determined in the framework of possible experimental standard deviations $\Delta_{\mathrm{N}}= \pm 0.035 \AA$ and $\Delta_{\mathrm{T}}=$ $\pm 0.06 \AA[20,21]$.

In the direction [001] (perpendicular to BL surface) just normal relaxation was detected between the first two planes $U_{\mathrm{N}}(1,2)=-0.02 \AA$, which corresponds to strain component $\epsilon_{\mathrm{N}}=0.014$. No surface relaxation was detected [20] between the planes (110), i.e. in the direction [110] (perpendicular to free surface LW in Fig. 1). Considering the experimental values of the basic elastic constants $C_{11}=2.33, C_{12}=1.35$, and $C_{44}=1.18 \times 10^{11} \mathrm{~N} \mathrm{~m}^{-2}$ for bcc iron at room temperature by $[22]$ and the coordination system by Fig. 1, i.e. ' $x_{1}=[001],{ }^{\prime} x_{2}=[110],{ }^{\prime} x_{3}=[110]$, the surface tension at the (001) surface can be estimated from the relations $\sigma_{1}={ }^{\prime} C_{11} \varepsilon_{1}+{ }^{\prime} C_{12} \varepsilon_{2}+{ }^{\prime} C_{13} \varepsilon_{3} ; \sigma_{2}={ }^{\prime} C_{21} \varepsilon_{1}$ $+{ }^{\prime} C_{22} \varepsilon_{2}+{ }^{\prime} C_{23} \varepsilon_{3} ; \sigma_{3}={ }^{\prime} C_{31} \varepsilon_{1}+{ }^{\prime} C_{32} \varepsilon_{2}+{ }^{\prime} C_{33} \varepsilon_{3}$ using $\varepsilon_{1}=\epsilon_{\mathrm{N}}=-0.014, \varepsilon_{3}=0$ in the [110] direction, $\sigma_{1}=\sigma_{\mathrm{N}}=0$ in the [001] direction perpendicular to the free surface (001) and the symmetry ' $C_{i j}={ }^{\prime} C_{j i}$.

The symmetric matrix of the transformed elastic constants ' $C_{i j}$ for our crystal orientation is given by the relations utilized in [15]: ' $C_{11}=C_{11},{ }^{\prime} C_{12}=$ $\mathrm{C}_{12},{ }^{\prime} C_{13}=C_{12},{ }^{\prime} C_{22}=\left(C_{11}+C_{12}+2 C_{44}\right) / 2=$
${ }^{\prime} C_{33},{ }^{\prime} C_{23}=\left(C_{11}+C_{12}-2 C_{44}\right) / 2,{ }^{\prime} C_{44}=\left(C_{11}-\right.$ $\left.C_{12}\right) / 2$, and ' $C_{55}={ }^{\prime} C_{66}=C_{44}$. The required numerical values are ' $C_{11}=2.33,{ }^{\prime} C_{22}=3.02$, and ' $C_{12}=$ $1.35 \times 10^{11} \mathrm{~N} \mathrm{~m}^{-2}$. By this method, we obtain $\varepsilon_{2}=$ 0.024 , further $\sigma_{2}=5.42 \mathrm{GPa}$ in the [110] direction and $\sigma_{3}=-0.3 \mathrm{GPa}$ in the [110] direction.

The component $\sigma_{2}=5.42 \mathrm{GPa}$ represents the surface tension along the free (001) surface by experimental data on surface relaxation. We may use it to estimate the expected shear stress and dislocation acceleration at the free surface (001). The nominal tension stress at time step 11880 from external loading in MD is $\sigma_{0}=4.38 \mathrm{GPa}$. The resulting tension stress in the [110] direction at the right free surface BL in Fig. 1 is $\sigma_{y}=4.38 \mathrm{GPa}+5.42 \mathrm{GPa}=9.80 \mathrm{GPa}$. The last relation shows that the surface tension $(5.42 \mathrm{GPa})$ coming from experimental data on surface relaxation is comparable with the nominal tension stress (4.38 GPa) coming from external loading in MD. So, the resulting shear stress in the $\langle 111\rangle\{112\}$ slip system at the right free surface $\mathrm{BL}$ should be given by relation $\tau=\sin \alpha \cos \alpha \sigma_{y}=0.47 \times 9.80 \mathrm{GPa}=4.61 \mathrm{GPa}$.

The range of interatomic forces is at least up to the second neighbors, due to the mechanical stability of the bcc lattice, so the atoms at the position $44 b, 45 b$, and $46 b$ are hit by the surface relaxation. The atom 45 already has the full number of interactions in the [110] direction, i.e., the elastic constant ' $C_{11}=3.02 \times 10^{11} \mathrm{~N} \mathrm{~m}^{-2}$ should be valid in this case, and a reasonable approach is an idea that the shear stress $\tau$ acts on the distance $2 b$, i.e., between the atoms $44 b-46 b$, which is in the range of the interaction $a_{0}$ up to the second neighbors. Thus, the resulting slip force is $F_{\mathrm{s}}=\tau 2 b=2.288 \mathrm{~N} \mathrm{~m}^{-1}$ and the resulting driving force at the free surface is $F=F_{\mathrm{s}}-F_{\mathrm{PN}}=(2.288-$ $0.057) \mathrm{N} \mathrm{m}^{-1}=2.231 \mathrm{~N} \mathrm{~m}^{-1}$.

If we suppose that $F(11880)=F=2.231 \mathrm{~N} \mathrm{~m}^{-1}$ then, according to the Newton's equations of motion mentioned above, we may write: $\Delta V_{\text {disl }}=V_{\text {disl }}(11900)$ - $V_{\text {disl }}(11880)=F(11880) 20 h / M_{1}=1950 \mathrm{~m} \mathrm{~s}^{-1}$, where $\Delta V_{\text {disl }}$ is the acceleration of a thought (hypothetical, suppositional) dislocation at the free surface (001) caused by the initial surface tension. Further, the final speed of the dislocation arriving to free surface (001) is estimated to be: $V_{\text {disl }}(11900)=$ $2210+1950=4160 \mathrm{~m} \mathrm{~s}^{-1}$, above $C_{\mathrm{T}}$. The surface tension ( $5.42 \mathrm{GPa}$ ) itself (without the nominal shear stress) leads to $\tau=2.5474 \mathrm{GPa}$, further to $F_{\mathrm{s}}=$ $\tau 2 b=1.2648 \mathrm{~N} \mathrm{~m}^{-1}$ and to an acceleration $\Delta V_{\text {disl }}=$ $F_{\mathrm{s}} 20 h / M_{1}=1106 \mathrm{~m} \mathrm{~s}^{-1}$ (considering a shorter range of interactions $1 b$, we obtain $553 \mathrm{~m} \mathrm{~s}^{-1}$ ).

This estimate follows from the experiments on surface relaxation at room temperature. Note that the possible error of the estimate may be significant due to the significant experimental errors mentioned above and due to different elastic properties in the surface layers in comparison with the bulk crystal. 
Table 2. Initial stress $S(0)$ after surface relaxation in MD at the individual atoms lying in the positions $42 b, 43 b, 44 b$, $45 b$, and $46 b$ (free surface) in the slip system $<111>\{112\}$, the total shear stress $\tau=\tau_{\mathrm{A}}+\tau(0)$ in units of GPa, further the slip $F_{\mathrm{s}}=\tau b$ and driving force $F=F_{\mathrm{s}}-F_{\mathrm{PN}}$ in units of $\mathrm{N} \mathrm{m}^{-1}$

\begin{tabular}{cccccccc}
\hline$r / b$ & $S_{x}(0)$ & $S_{y}(0)$ & $S_{z}(0)$ & $\tau(0)$ & $\tau(r)$ & $F_{\mathrm{s}}(r)$ & $F(r)$ \\
\hline 42 & -0.1217 & 0.2364 & -1.2840 & 0.1688 & 2.2335 & 0.5545 & 0.4975 \\
43 & -0.0796 & 0.2476 & -1.3130 & 0.1538 & 2.2185 & 0.5507 & 0.4937 \\
44 & -0.0460 & 0.3657 & -1.2110 & 0.1941 & 2.2588 & 0.5608 & 0.5038 \\
45 & -0.0215 & -0.4150 & -2.110 & -0.1849 & 1.8748 & 0.4654 & 0.4084 \\
46 & -0.0067 & 3.1060 & 1.4320 & 1.4673 & 3.5320 & 0.8782 & 0.8212 \\
\hline
\end{tabular}

The concept of the interplanar stress [4] in the atomistic lattice does not need the elastic constants and implicitly includes the changed elastic properties by means of the changed interatomic interactions at the free surface after the surface relaxation and, as well, the model satisfies the conditions of the free surface (see Fig. 2b), similar to nature.

To explain the role of the initial stress after surface relaxation in MD we performed stress calculations on the atomistic level in the neighborhood of the key atomic positions of the lower dislocation core lying at the distance $42 b, 43 b, 44 b, 45 b$, and $46 b$ (surface atom) from the crack tip in the slip system [111] $(\overline{1} 1 \overline{2})$; see Figs. 5a, 9. The results of these stress calculations are given in Table 2 .

Unlike the surface stress from experimental data discussed above, the initial surface stress in MD is of somewhat longer range (about $2-3 a_{0}$ ), and there is a different distribution of the initial stress between the $y$ - and $z$-stress components. This is caused by the presence of the long edge crack close to the free surface (see Figs. 1, 2) and it may also be influenced by the atomistic model in use as follows from [21, 23].

Nevertheless, the behavior in MD seems to be realistic: the component $S_{x}(0)$ practically vanishes at the surface atom $46 b$, while $S_{y}(0)$ reaches the maximum here and no shear relaxation is monitored in MD at the free surface (001), in agreement with experiments [20] on surface relaxation in bcc iron. The initial shear stress $\tau(0)$ in Table 2 was calculated from a general relation $\tau=\sqrt{2}\left(\sigma_{Y}-\sigma_{X}\right) / 3+\sigma_{X Y} / 3$ for the slip systems $<111>\{112\}$ at the crack (110)[110] considering $\sigma_{X Y}=0, \sigma_{Y}=S_{y}(0)$ and $\sigma_{X}=S_{x}(0)$.

The resulting shear stress is given as $\tau=\tau_{\mathrm{A}}+$ $\tau(0)$, where $\tau_{\mathrm{A}}=2.065 \mathrm{GPa}$ comes from the applied external loading at time step $11880\left(\tau_{\mathrm{A}}=0.47 \sigma_{0}\right)$, when the dislocation core is in the position $42-43 b$ (see Fig. 9 and Table 1). At this moment $(t=11880 h)$, the total driving force acting on the dislocation core in the surface region is given by Tab. 2, i.e. $F(11880)$ $=\sum F\left(r_{i}\right)$, and the Newton's equation of motion of the core can be written as

$$
M_{1} \Delta V_{\mathrm{disl}} / \Delta t=\sum F\left(r_{i}\right)
$$

$$
\sum F\left(r_{i}\right)=\sum\left(F_{\mathrm{s}}\left(r_{i}\right)-F_{\mathrm{PN}}\right)
$$

which means that $\Delta V_{\text {disl }}=V_{\text {disl }}(11900)-V_{\text {disl }}(11880)$ $=20 h / M_{1} \sum F\left(r_{i}\right)$.

If we suppose that at time step 11880 the dislocation core is at the position $42 b, \sum F\left(r_{i}\right)=F(42)+$ $F(43)+F(44)+F(45)+F(46)=2.7246 \mathrm{~N} \mathrm{~m}^{-1}$ by Table 2, further $\Delta V_{\text {disl }}=2382 \mathrm{~m} \mathrm{~s}^{-1}$ and the final transonic velocity is $V_{\text {disl }}(11900)=2210 \mathrm{~m} \mathrm{~s}^{-1}+$ $2382 \mathrm{~m} \mathrm{~s}^{-1}=4592 \mathrm{~m} \mathrm{~s}^{-1}=1.53 C_{\mathrm{T}}$. It lies within the interval $3724-7447 \mathrm{~m} \mathrm{~s}^{-1}$ following from MD-results in Fig. 9 and the dislocation reaches the free surface $(r=46 b)$ near the time step 11900 in agreement with MD (by about $1.6 \mathrm{~h}$ later).

If we assume that, at the time step 11880 , the dislocation core is at position $43 b$, the total driving force is $\sum F\left(r_{i}\right)=F(43)+F(44)+$ $F(45)+F(46)=2.2271 \mathrm{~N} \mathrm{~m}^{-1}$, further $\Delta V_{\text {disl }}=$ $20 h / M_{1} \sum F\left(r_{i}\right)=1947 \mathrm{~ms}^{-1}$ and the final transonic velocity is $V_{\text {disl }}(11900)=2210 \mathrm{~m} \mathrm{~s}^{-1}+$ $1947 \mathrm{~m} \mathrm{~s}^{-1}=4157 \mathrm{~m} \mathrm{~s}^{-1}=1.38 C_{\mathrm{T}}$. This is a more realistic MD result for two reasons:

i. The distribution of the individual driving forces $F\left(r_{i}\right)$ in Table 2 indicates that the dislocation should accelerate at a very close distance from the free surface;

ii. The lower transonic velocity $1.38 C_{\mathrm{T}}$ better complies with the Mach angle following from the reconstruction of the AE patterns in Fig. 10. The dislocation speed $1.38 C_{\mathrm{T}}$ also means that it reaches the free (001) surface at time step 11 898, i.e., before 11 $900 h$, which is in a qualitative agreement with Fig. 7 and with the results following from the experiments on surface relaxation.

To model a more detailed distribution of the velocities in the surface region, one must take into account the interplanar [4] character of the stress calculations in the present study. This means that the last values in Table 2 concern the interplanar distance between the positions $45 b-46 b$ and the first values are valid for the positions between $42 b-43 b$, etc. The velocities inside these intervals are constant and they can be determined in a standard way by means of weighted 
functions from the relations:

$$
\begin{gathered}
\Omega_{i}=F\left(r_{i}\right) / \sum F\left(r_{i}\right), \\
\sum \Omega_{i}=1, \\
\Delta V_{i}=\Omega_{i} \Delta V_{\mathrm{disl}}, \\
\Delta V_{\mathrm{disl}}=1947 \mathrm{~m} \mathrm{~s}^{-1}, \\
V_{\mathrm{disl}}(i)=V_{\mathrm{disl}}(i-1)+\Delta V_{i} .
\end{gathered}
$$

In such a way we obtain the distribution of the velocities in the surface region, visualized in Fig. 12a:

\begin{tabular}{cccc}
\hline$r / b$ & $\Omega_{i}$ & $\Delta V_{i}$ & $V_{\text {disl }}(i)$ \\
\hline $42-43$ & 0.2217 & 432 & 2642 \\
$43-44$ & 0.2262 & 440 & 3082 \\
$44-45$ & 0.1834 & 357 & 3439 \\
$45-46$ & 0.3687 & 718 & 4157 \\
\hline
\end{tabular}

With the initial constant velocity $2210 \mathrm{~m} \mathrm{~s}^{-1}$, the position $42 b$ is reached at time step 11870 . The next position $43 b$ is reached during $\Delta t=1 b / V_{\text {disl }}(i)=$ $1 b / 2642 \approx 9$ h, i.e. at time step 11879 . Following this scheme, we obtain the time dependence $r / b$ vs. $\Delta t / \mathrm{h}$ shown in Fig. 12 b.

The initial constant velocity $2210 \mathrm{~m} \mathrm{~s}^{-1}$ in the region 18-42b in Figs. 9, 12 can be explained using the results presented in Figs. 4c, 5c. Figure 4c shows that the velocity of dislocation generation at the time interval 11500 (unstable position $b / 2$ ) - 11600 (complete $b$ ) is $V_{\mathrm{gnr}}=206 \mathrm{~m} \mathrm{~s}^{-1}$ as mentioned above. Figure $5 \mathrm{c}$ illustrates that at time step 11600 the stress concentration at the crack front after dislocation emission decreases approximately to a nominal level of external loading $\left(\sigma_{0}(11600)=4.28 \mathrm{GPa}\right)$, i.e., the slip force in the slip system $\langle 111\rangle\{112\}$ comes just from external loading, which is $F_{\mathrm{S}}(11600)=0.4994$ $\mathrm{N} \mathrm{m}^{-1}$ as mentioned above. By the Newton's equation of motion, we may write $V_{0}(11600)-V(11500)=$ $100 h / M_{1}\left(F_{\mathrm{S}}(11600)-F_{\mathrm{PN}}\right)=1936 \mathrm{~m} \mathrm{~s}^{-1}$ and taking $V(11500)=V_{\text {gnr }}$ we obtain $V_{0}=2142 \mathrm{~m} \mathrm{~s}^{-1}$, which complies well with the average value $2210 \pm 407 \mathrm{~m} \mathrm{~s}^{-1}$ following from Fig. 9.

One may see that the surface stress, following either from experimental data on surface relaxation in bcc iron or from present MD simulations in the 3D iron crystal (Fig. 1), can explain the acceleration of dislocations at the short distance from the relaxed (001) free surfaces.

All the effects mentioned above are reflected by the global energy balance in MD during the first emission shown in Fig. 4a and presented in more detail in Table 1 . The physical reason for dislocation attraction to the free surface is the possibility to decrease the potential (strain) energy in the system by disappearing of the dislocation at the free surface - see the upper curve in Fig. 4a. So, the driving force for dislocation motion can also be approximated from the global energy balance as

$$
F_{\text {disl }}=-\Delta E P O T / \Delta A
$$

where $\Delta A=\Delta r B$ is the area swept by the moving dislocation. Here, $\Delta r$ is the displacement of the dislocation line in the direction $\langle 111\rangle$ of the Burgers vector on a slip plane $\{112\}$ and $B=30 a_{0} \sqrt{2} / 2$ is the length of the dislocation line in the [110] direction. The values $F_{\text {disl }}$ are inserted in Table 1 , and they are related again to Fig. 9 and to the time step 11600 , i.e., $\triangle E P O T=$ $\operatorname{EPOT}(t)-\operatorname{EPOT}(11600 h), \Delta r=r(t)-r(11600 h)$.

This approximation implicitly includes all the members mentioned above, i.e., the attractive surface forces, lattice resistance $F_{\mathrm{PN}}$, the slip forces $F_{\mathrm{S}}$ from external loading or induced by the crack, as well as the contribution from the surface tension. Simplification of the evaluation is an assumption of the straight edge dislocation emitted by the crack in $3 \mathrm{D}$.

According to Newton's law concerning the impulse of a mass $m$ point with a driving force $F(t)$ :

$$
\begin{aligned}
& m v_{1}-m v_{0}= \\
& \int_{t_{0}}^{t_{1}} F(t) \mathrm{d} t, \text { or for the discrete time sampling } \\
& m v_{1}-m v_{0}=\sum_{t_{0}}^{t_{1}} F(t) \Delta t
\end{aligned}
$$

one may expect in the interval $<11600 h, 11900 h>$ an increment in dislocation velocity given by Eq. (12):

$$
\begin{aligned}
& \Delta V_{\text {disl }}=V_{\text {disl }}(11900 h)-V_{\text {disl }}(11600 h)= \\
& \left(20 h / M_{1}\right) \sum F_{\text {disl }}(t),
\end{aligned}
$$

where the sum is taken over all the data $F_{\text {disl }}$ presented in Table 1.

This gives an increment of the dislocation velocity $1836 \mathrm{~m} \mathrm{~s}^{-1}$ and the final dislocation velocity at the free surface $V_{\text {disl }}(11900)=1836+2210=4046$ $\mathrm{m} \mathrm{s}^{-1}=1.35 C_{\mathrm{T}}$. The model well describes the time dependence in Fig. 9 up to time step $11820(\Delta t / \mathrm{h}=$ 220) where the dislocation begins to slowly accelerate by the global energy balance up to the final velocity $4046 \mathrm{~m} \mathrm{~s}^{-1}$ at time step 11900 . Note that the energy concept and modeling by finite element method were used [24] to evaluate the image forces without the fictitious image dislocations. 
$\left(\mathrm{E}_{\text {kin }}\left(\mathrm{I}_{\mathrm{i}}\right) / 10^{-22}\right)^{1 / 2}$, double layer=8
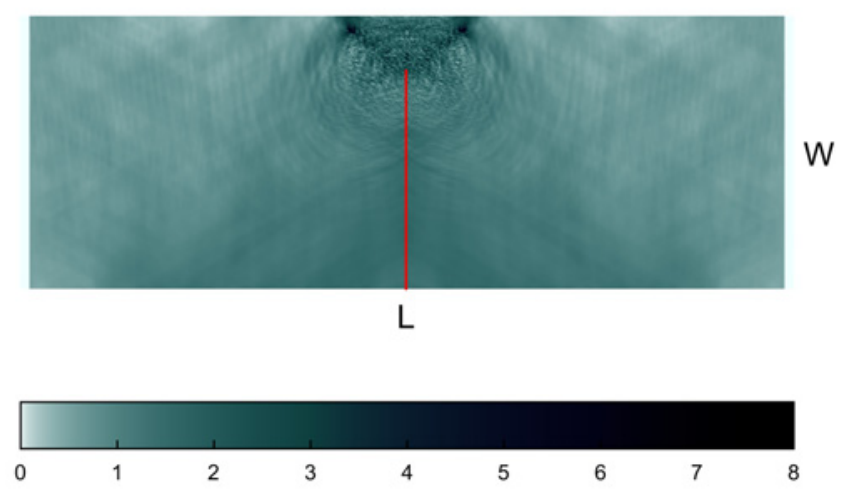

$\left(E_{\text {kin }}\left(I_{\mathrm{i}}\right) / 10^{-22}\right)^{1 / 2}$, double layer $=110$

(b)

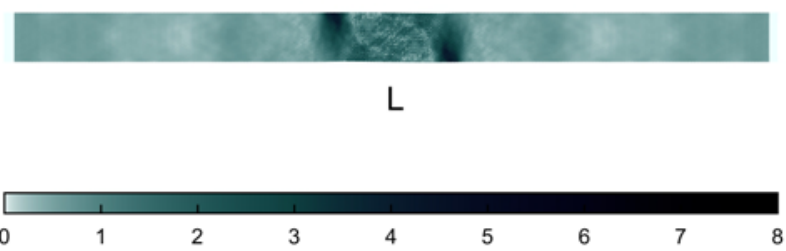

Fig. 11. The character of acoustic emission from the first dislocation emission under higher loading rate $0.034 K_{\mathrm{G}} / \mathrm{ps}$, time $t=4000 h$, i.e. $K_{\mathrm{A}}(t)=t *\left(0.034 K_{\mathrm{G}} / \mathrm{ps}\right)=1.36 K_{\mathrm{G}}$ : (a) acoustic emission in the middle planes (110); (b) AE signal on the upper LB surface (001).

For comparison, the radiation-free velocity following from continuum analysis in [11], determined from the relation for orthotropic solids with in-plane displacements $u_{1}, u_{2}$ and utilizing the elastic constants ' $C_{i j}$ above for the evaluation of the experimental surface relaxation is $V_{\mathrm{rf}}=3886 \mathrm{~m} \mathrm{~s}^{-1}$. A similar value $V_{\mathrm{rf}}=3891 \mathrm{~m} \mathrm{~s}^{-1}$ is obtained with elastic constants valid $[5,6]$ at low temperatures for our MD simulations and their potential in use.

As mentioned in the Introduction, dislocation emission also occurs under higher loading rates in mode I, e.g., for $0.034 K_{\mathrm{G}} /$ ps presented in Fig. 11. In this case, the dislocation emission is less pronounced in the global energy balance, due to more significant influence of the dynamic effects such as scattering of the loading waves on crack faces, excitation of eigen vibrations, expansion of the pressure unloading waves from the corners (so-called von Schmidt waves in isotropic continuum), etc.

The last dynamic effects are visible in Fig. 11 and they are discussed in more detail in [6]. Nevertheless, the kinetics of dislocation motion (evaluated in the same way as above) is similar as presented for the relatively slow loading rate $0.010 K_{\mathrm{G}} / \mathrm{ps}$. Under higher loading rate $0.034 K_{\mathrm{G}} / \mathrm{ps}$, the emitted dislocations in the bulk of the crystal move with an average subsonic velocity of about $2000 \mathrm{~m} \mathrm{~s}^{-1}$ and accelerate not before a distance $R=5 b$ from the right free surface. At this distance, the very narrow surface region in MD is penetrated with transonic velocities 3 103-6 $206 \mathrm{~m} \mathrm{~s}^{-1}$. This means that the acceleration of dislocations in surface layers does not depend on external loading rate.

Motion of the curved dislocations in 3D (such as in Fig. 3) is a complex process: it can be accelerated via kink edge segments [25] or hindered via screw segments with a more complex (3D) core structure and the larger Peierls stress $\tau_{\mathrm{PN}}$ in comparison with the edge dislocations of planar character $[19,26]$. The screw segments may create the jogs producing vacancies [3], which also hinders the motion, the dislocation speed may also differ due to the various stress conditions in the middle of the sample and nearby the free surfaces, etc.

Utilizing the global energy balance from MD to assess the speed of the edge dislocation segments (at $B / 2$ ) is thus a simplification of the $3 \mathrm{D}$ problem and represents a rather lower estimate for the acceleration of dislocations toward the free surface. For the local description of the motion of the edge dislocation segments in the middle of the crystal, a more realistic estimate offers the theory that acceleration of edge dislocations at the free surface (001) comes from the short ranged surface stress in bcc iron.

Recent continuum analysis [27] of surface waves in anisotropic medium shows that a transonic surface wave, with the two components polarized perpendicular and parallel to the (001) surface, can be accommodated in cubic crystals [27, 28], unlike the isotropic medium. Such a mode can be used in our case for the fast edge dislocations (Figs. 5-7) with Burgers vectors $\langle 111\rangle$ lying on a (110) plane to penetrate the free (001) LB surface. The Burgers vectors determining the motion of dislocation cores are composed, in our case, from the one [001] component (perpendicular to LB) and the second $\langle\overline{1} 10\rangle$ component (parallel to LB).

Figure 4 in this paper illustrates that the generation of the dislocations by the crack can be very fast with disturbance time of about $100 \mathrm{~h}$, i.e., with an operating period of about 1 picosecond. This is not directly accessible for experimental observation, e.g. via in-situ electron microscope, similar to the fast dislocation motion presented in Fig. 12. Since the generation and motion of fast dislocations play an important role in technical (engineering) applications, e.g., such as shock loading of solids [29], continuous attention is devoted to various aspects of the fast dislocation motion both in the theoretical studies (e.g., [30-32]) and in atomistic studies via molecular dynamics (e.g., $[33,34])$. Such studies contribute to an understanding of the process and create a base for proposals of verification experiments in future, e.g., via the detection of $\mathrm{AE}$ signal from the fast dislocation motion or via measurements of dislocation density [29] and their post-processing analysis. 

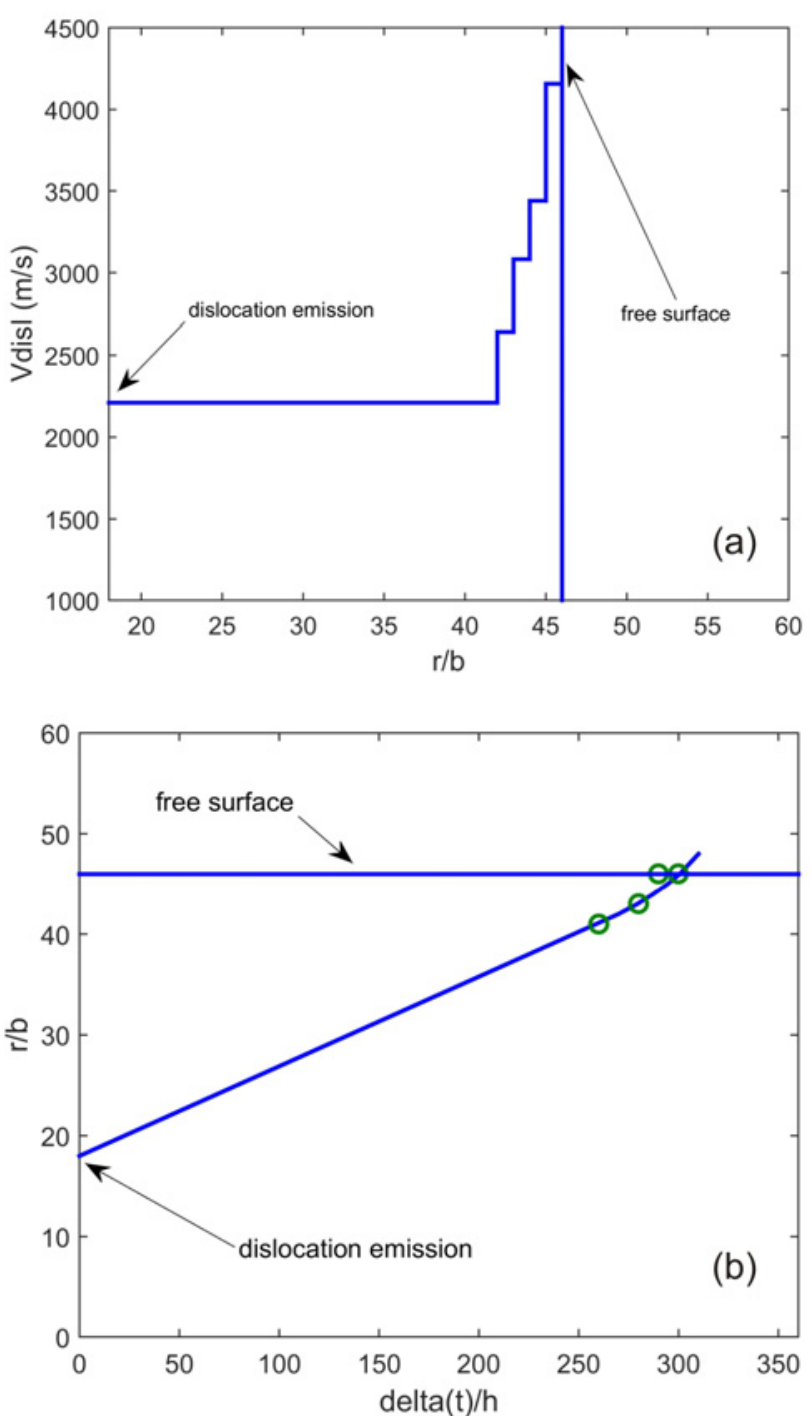

Fig. 12. The results from the model including the initial stress after surface relaxation: (a) course of the dislocation velocity toward the free surface; (b) the curved line shows the time dependence of the dislocation core positions by the model, the circles (o) represent MD data at the free surface.

In this study, the substantial part of the fast dislocation motion is covered by the high nominal tension stress $\sigma_{0}$ needed for dislocation emission from the crack of nano-scale length $a=25.2252 \mathrm{~nm}$ since $\sigma_{0} \sim K_{\text {disl }}(\pi a)^{-1 / 2}$. In actual experimental fracture specimens, the critical stress $\sigma_{0}$ needed for dislocation emission is far lower due to much larger values of the initial crack length $a$, and so the dislocation motion may not reach the transonic regime, as in the present MD study. However, the contribution from the surface stress can cause some acceleration of dislocations toward the free surface.

The surface stress itself (without any external loading) may cause a significant increment in dislocation velocity in the [001] direction (of about $400 \mathrm{~m} \mathrm{~s}^{-1}$ by the present study), which complies with experimental data on surface relaxation in bcc iron. This concerns just very near vicinity of the free surface, since the surface stress is short ranged in the [001] direction: 2$3 a_{0}$ by the present MD study, about 1 lattice parameter $a_{0}$ by the experiments on surface relaxation [20] and about 5 interplanar distances $d_{001}$ (i.e., $2.5 a_{0}$ ) by quantum mechanical treatment [23] of $\{001\}$ surfaces in bcc iron.

Note that the range of surface relaxation (and consequently of the surface stress) differs in the individual crystallographic directions of bcc iron (see [20, 21, 23]) due to the anisotropy.

This study indicates that the disappearance of dislocations at the free crystal surfaces may be assisted by the initial surface tension after the surface relaxation. As mentioned in [23], surface relaxation is also important for other phenomena, such as adhesion or adsorption of impurities at free surfaces, etc.

\section{Summary}

Dislocation emission from edge crack $a / W=0.8$ embedded in a $3 \mathrm{D}$ bcc iron crystal was studied via $\mathrm{MD}$ by direct graphical treatment of the atomistic configurations, further via mapping of the local kinetic energies of individual atoms (revealing the acoustic emission from dislocations) and also from the global energy balance.

The graphical treatment of MD results shows that the edge dislocation segments in the middle of the crystal after the emission move on the slip systems $\langle 111\rangle\{112\}$ with a subsonic velocity up to a very close distance from the free surface (001) where they accelerate and penetrate the surface layers in transonic or supersonic regime.

An analysis of the global energy balance and acoustic emission from the moving dislocations has shown that dislocation motion in MD nearby the free surface (001) can be accelerated to the transonic regime (not supersonic), i.e., just above the velocity $C_{\mathrm{T}}$ of the transversal shear waves in the $\langle 111\rangle$ direction.

Additional (complementary) stress calculations on the atomistic level reveal the reason: the dislocations in MD accelerate to transonic regimes at a close distance from the free surface, due to short ranged surface tension. It complies with experimental data on surface relaxation in bcc iron.

The transonic regime $\left(V_{\text {disl }}=1.35 C_{\mathrm{T}}-1.38 C_{\mathrm{T}}\right)$ following from this study does not concern the dislocations in macroscopic experimental specimens under a low external loading. Here, the surface stress itself (without any external loading) may only cause an increment in dislocation velocity (of about $400 \mathrm{~m} \mathrm{~s}^{-1}$ by this study). It concerns a very short distance $1-3$ lat- 
tice parameter from the free surface (001). The range of surface relaxation (and consequently the range of surface stress) in bcc iron differs in the individual crystallographic directions.

This study indicates that the existence of initial surface tension can facilitate the disappearing of dislocations at the free crystal surfaces.

Further studies, focused on the development of a reliable description of interatomic forces both in the bulk crystal and at the free surfaces (based on quantum mechanics) and more precise experimental data on surface relaxation and on AE signal at free surfaces are needed to verify the acceleration of dislocations nearby the free surfaces.

\section{Acknowledgements}

The work was supported by the Institute Research Program RVO:61388998 and by the projects of the Czech Science Foundation GACR 17-22615S and GACR 17-12925S. The work of P. Hora was supported by the European Regional Development Fund under Grant No.CZ.02.1.01/0.0/0.0/15_003/0000493 (Centre of Excellence for Nonlinear Dynamic Behavior of Advanced Materials in Engineering).

\section{References}

[1] Koehler, J. S.: Phys. Rev., 60, 1941, p. 397. doi:10.1103/PhysRev.60.397

[2] Gurrutxaga-Lerma, B., Balint, D. S., Dini, D., Sutton, P.: Proc. R. Soc. A, 471, 2015, p. 0433. doi:10.1098/rspa.2015.0433

[3] Hirth, J. P., Lothe, J.: Theory of Dislocations. New York, Wiley 1982. ISBN 0471091251.

[4] Machová, A.: Modelling Simul. Mater. Sci. Eng., 9, 2001, p. 327. doi:10.1088/0965-0393/9/4/306

[5] Ackland, G. J., Bacon, D. J., Calder, A. F., Harry, T.: Phil. Mag. A, 75, 1997, p. 713. doi:10.1080/01418619708207198

[6] Eshelby, J. D.: Proc. Phys. Soc. Lond. A, 62, 1949, p. 307. doi:10.1088/0370-1298/62/5/307

[7] Gumbsch, P., Gao, H.: Journal of Computer-Aided Materials, 6, 1999, p. 137. doi:10.1023/A:1008789505150

[8] Gumbsch, P., Gao, H.: Science, 283, 1999, p. 965. doi:10.1126/science.283.5404.965

[9] Jin, Z., Gao, H., Gumbsch, P.: Phys. Rev. B, 77, 2008, p. 094303. https://link.aps.org/doi/10.1103/PhysRevB.77. $\underline{094303}$

[10] Cerv, J., Landa, M., Machová, A.: Scripta Materialia, 43, 2000, p. 423. doi:10.1016/S1359-6462(00)00456-5

[11] Gao, H., Huang, Y., Gumbsch, P., Rosakis, A. J.: Journal of the Mechanics and Physics of Solids, 47, 1999, p. 1941. doi:10.1016/S0022-5096(98)00126-4
[12] Machová, A., Beltz, G. E.: Kovove Mater., 36, 1998, p. 135.

[13] Fett, T.: Stress Intensity Factors, T-Stresses, Weight Functions. Karlsruhe, Universitätsverlag Karlsruhe 2008, p. 228. ISBN: 978-3-86644-235-1.

[14] Rice, J. R.: J. Mech. Phys. Solids, 40, 1992, p. 239. doi:10.1016/S0022-5096(05)80012-2

[15] Beltz, G. E., Machová, A.: Modelling Simul. Mater. Sci. Eng., 15, 2007, p. 65. doi:10.1088/0965-0393/15/2/005

[16] Spielmanová, A., Machová, A., Hora, P.: Acta Materialia, 57, 2009, p. 4065 . doi:10.1016/j.actamat.2009.04.048

[17] Eshelby, J. D.: Acta Metallurgica, 1, 1953, p. 251. doi:10.1016/0001-6160(53)90099-6

[18] Pellegrini, Y. P.: Wave Motion, 68, 2017, p. 128. doi:10.1016/j.wavemoti.2016.09.006

[19] Vitek, V.: Crystal Lattice Defects, 5, 1974, p. 1.

[20] Legg, K. O., Jona, F. Jepsen, D. W.: Journal of Physics C: Solid State Physics, 10, 1977, p. 937. doi:10.1088/0022-3719/10/7/005

[21] Machová, A., Mach, R.: Surface Science, 211-212, 1989, p. 125. doi:10.1016/0039-6028(89)90762-0

[22] Rayne, J. A., Chandrasekhar, B. S.: Phys. Rev., 122, 1961 , p. 1714. https://link.aps.org/doi/10.1103/PhysRev.122.1714

[23] Spencer, M. J. S., Hung, A., Snook, I. K., Yarovsky, I.: Surface Science, 513, 2002, p. 389. doi:10.1016/S0039-6028(02)01809-5

[24] Khanikar, P., Kumar, A., Subramaniam, A.: Phil. Mag., 91, 2011, p. 730. doi: $10.1080 / 14786435.2010 .529089$

[25] Salje, E. K. H., Wang, X., Ding, X., Scott, J. F.: Advanced Functional Materials, 27, 2017, p. 1700367. doi:10.1002/adfm.201700367

[26] Yamaguchi, M., Vitek, V.: J. Physics F: Metal Physics, 5, 1975, p. 11. doi:10.1088/0305-4608/5/1/005

[27] Favretto-Cristini, N., Komaditich, V., Garcione, J., Cavallini, F.: Ultrasonics, 51, 2011, p. 653. doi:10.1016/j.ultras.2011.02.007

[28] Chadwick, P., Wilson, N. J.: Proc. Roy. Soc. London: Mathematical and Physical Sciences, 438, 1992, p. 225. doi:10.1098/rspa.1992.0104

[29] Hahn, E. N., Zhao, S., Bringa, E. M., Meyers, M. A.: Scientific Reports, 6, 2016, p. 1. https://www.nature.com/articles/srep26977

[30] Huang, S., Markenscoff, X.: International Journal of Engineering Science, 49, 2011, p. 1461. doi:10.1016/j.ijengsci.2011.03.009

[31] Lazar, M., Pellegrini, Y. M.: Journal of the Mechanics and Physics of Solids, 96, 2016, p. 632. doi:10.1016/j.jmps.2016.07.011

[32] Shiroky, I. B., Gendelman, O. V.: Chaos, 28, 2018, p. 023104. doi:10.1063/1.5009098

[33] Burberry, N., Das, R., Ferguson, W. G.: Computational Materials Science, 124, 2016, p. 259. doi:10.1016/i.commatsci.2016.08.001

[34] Verschueren, J., Gurrutxaga-Lerma, B., Balint, D. S., Sutton, A. P., Dini, D.: Physical Review Letters, 121, 2018, p. 145502. doi:10.1103/PhysRevLett.121.145502 